\title{
Prioritized Conditional Imperatives: Problems and a New Proposal ${ }^{\star}$
}

\author{
Jörg Hansen \\ University of Leipzig, Institut für Philosophie \\ 04107, Leipzig, Beethovenstraße 15, Germany \\ jhansen@uni-leipzig.de
}

\begin{abstract}
The sentences of deontic logic may be understood as describing what an agent ought to do when faced with a given set of norms. If these norms come into conflict, the best the agent can be expected to do is to follow a maximal subset of the norms. Intuitively, a priority ordering of the norms can be helpful in determining the relevant sets and resolve conflicts, but a formal resolution mechanism has been difficult to provide. In particular, reasoning about prioritized conditional imperatives is overshadowed by problems such as the 'order puzzle' that are not satisfactorily resolved by existing approaches. The paper provides a new proposal as to how these problems may be overcome.
\end{abstract}

Keywords: deontic logic, default logic, priorities, logic of imperatives

\section{Drinking and Driving}

Imagine you have been invited to a party. Before the event, you become the recipient of various imperative sentences:

(1) Your mother says: if you drink anything, then don't drive.

(2) Your best friend says: if you go to the party, then you do the driving.

(3) Some acquaintance says: if you go to the party, then have a drink with me.

Suppose that as a rule you do what your mother tells you - after all, she is the most important person in your life. Also, the last time you went to a party your best friend did the driving, so it really is your turn now. You can enjoy yourself without a drink, though it would be nice to have a drink with your acquaintance - your best friend would not mind if you had one drink, and your acquaintance does not care that you may be driving - but your mother would not approve of such a behavior. Making up your mind,

(4) You go to the party.

I think it is quite clear what you must do: obey your mother and your best friend, and hence do the driving and deny your acquaintance's request. However, it is not so clear what formal algorithm could explain this reasoning.

* I am grateful to Lou Goble, John F. Horty and Leon van der Torre for helpful comments and discussions in preparing this paper.

Dagstuhl Seminar Proceedings 07122

Normative Multi-agent Systems

http://drops.dagstuhl.de/opus/volltexte/2007/914 
An example of a similar form was first employed in epistemic logic, ${ }^{1}$ and has been termed the 'order puzzle' (cf. Horty [22]). For the epistemic version, consider the following sentences:

(5) You remember from physics: if you are in a car, lightning won't strike you.

(6) The coroner tells you: he was struck by lightning.

(7) Your neighbor says: he must have been drinking and driving.

Suppose that driving includes being in a car, that you firmly believe in what you remember from physics, that you believe that information by medical officers is normally based on competent investigation, and that you usually don't question your neighbor's observations, but think that sometimes she is just speculating. It seems quite clear what happens: you keep believing what you remember from school, and don't doubt what the coroner told you, but question your neighbor's information, maybe answering: "This can't be true, as the authorities found he was struck by lightning, and you can't be struck by lightning in a car".

In both cases, the problem as to how the underlying reasoning can be formally reconstructed seems so far unsolved. Both involve a ranking, or priority ordering, of the sentences involved. Concentrating on the imperative side of things, in what follows, I will consider various proposals from the literature that have been put forward to explain the reasoning about such prioritized conditionals, discuss their strengths and weaknesses in relation to problems such as the one above, and finally propose a fresh solution that solves the problem.

\section{Formal Preliminaries}

To formally discuss problems such as the one presented above, I shall use a simple framework: let $I$ be a set of objects, they are meant to be (conditional) imperatives. Two functions $g$ and $f$ associate with each imperative an antecedent and a consequent - these are sentences from the language of a basic logic that here will be the language $\mathscr{L}_{P L}$ of propositional logic. ${ }^{2} g(i)$ may be thought of as describing the 'grounds', or circumstances in which the consequent of $i$ is to hold, and $f(i)$ as associating the sentence that describes what must be the case if the imperative $i$ is satisfied, its 'deontic focus' or 'demand'. ${ }^{3}$ In accordance with tradition (cf. Hofstadter and McKinsey [20]), I write $A \Rightarrow ! B$ for an $i \in I$ with $g(i)=A$ and $f(i)=B$, and $! A$ means an unconditional imperative $\top \Rightarrow ! A$. Note

${ }^{1}$ Cf. Rintanen [36] p. 234, who in turn credits Brewka with its invention.

${ }^{2} \mathrm{PL}$ is based on a language $\mathscr{L}_{P L}$, defined from a set of proposition letters Prop $=$ $\left\{p_{1}, p_{2}, \ldots\right\}$, Boolean connectives ' $\neg$ ', ' $\wedge$ ', ' $\vee$ ', ' $\rightarrow$ ', ' $\leftrightarrow$ ' and brackets '(', ')' as usual. The truth of a $\mathscr{L}_{P L}$-sentence $A$ is defined recursively using a valuation function $v: \operatorname{Prop} \rightarrow\{1,0\}$ (I write $v \models A$ ), starting with $v \models p$ iff $v(p)=1$ and continuing as usual. If $A \in \mathscr{L}_{P L}$ is true for all valuations it is called a tautology. $P L$ is the set of all tautologies, and this set is used to define provability, consistency and derivability (I write $\Gamma \vdash_{P L} A$ ) as usual. $\top$ is an arbitrary tautology, and $\perp$ is $\neg \top$.

${ }^{3}$ In analogy to Reiter's default logic one might add a third function $e$ that describes exceptional circumstances in which the imperative is not to be applied. I will not address this additional complexity here. 
that $A \Rightarrow ! B$ is just the name for a conditional imperative that demands $B$ to be made true in a situation where $A$ is true - it is not an object that is assigned truth values. I write $m(i)$ for $\ulcorner g(i) \rightarrow f(i)\urcorner$ and call $m(i)$ the 'materialization' of $i$, as it represents the material implication that may be thought of as corresponding to the conditional imperative. For any $i \in I$ and $\Delta \subseteq I$, instead of $f(i), g(i)$, $m(i), f(\Delta), g(\Delta)$ and $m(\Delta)$, I may use the superscripted $i^{f}, i^{g}, i^{m}, \Delta^{f}, \Delta^{g}$ and $\Delta^{m}$ for better readability.

Let $\mathcal{I}$ be a tuple $\langle I, f, g\rangle$, let $W \subseteq \mathscr{L}_{P L}$ be a set of sentences, representing 'real world facts', and $\Delta \subseteq I$ be a subset of the imperatives: then we define

$$
\operatorname{Triggered}_{\mathcal{I}}(W, \Delta)=_{d f}\left\{i \in \Delta \mid W \vdash_{P L} g(i)\right\} .
$$

So an imperative $i \in \Delta$ is triggered if its antecedent is true given $W$. Tradition wants it that a conditional imperative can only be fulfilled or violated if its condition is the case. ${ }^{4}$ So I define:

$$
\begin{aligned}
& \text { Satisfied }_{\mathcal{I}}(W, \Delta)=_{d f}\left\{i \in \Delta \mid W \vdash_{P L} i^{g} \wedge i^{f}\right\}, \\
& \operatorname{Violated}_{\mathcal{I}}(W, \Delta)={ }_{d f}\left\{i \in \Delta \mid W \vdash_{P L} i^{g} \wedge \neg i^{f}\right\},
\end{aligned}
$$

An imperative in $\operatorname{Satisfied}_{\mathcal{I}}(W, \Delta)\left[\operatorname{Violated}_{\mathcal{I}}(W, \Delta)\right]$ is called satisfied [violated] given the facts $W$. It is of course possible that an imperative is neither satisfied nor violated given the facts $W$. If an imperative is triggered, but not violated, we call the imperative satisfiable:

$$
\text { Satisfiable }_{\mathcal{I}}(W, \Delta)=_{d f}\left\{i \in \operatorname{Triggered}_{\mathcal{I}}(W, \Delta) \mid W \nvdash_{P L} \neg i^{f}\right\} .
$$

Moreover, we define

$$
\text { Obeyable }_{\mathcal{I}}(W, \Delta)=_{d f}\left\{\Gamma \subseteq \Delta \mid \Gamma^{m} \cup W \nvdash_{P L} \perp\right\} .
$$

So a subset $\Gamma$ of $\Delta$ is obeyable given $W$ iff it is not the case that for some $\left\{i_{1}, \ldots, i_{n}\right\} \subseteq \Gamma$ we have $W \vdash_{P L}\left(i_{1}^{g} \wedge \neg i_{1}^{f}\right) \vee \ldots \vee\left(i_{n}^{g} \wedge \neg i_{n}^{f}\right)$ : otherwise we know that whatever we do, i.e. given any maxiconsistent subset $V$ of $\mathscr{L}_{P L}$ that extends $W \subseteq V$, at least one imperative in $\Gamma$ is violated. ${ }^{5}$ We speak of a conflict of imperatives when the triggered imperatives cannot all be satisfied given the facts $W$, i.e. when Triggered $_{\mathcal{I}}(W, \Delta)^{f} \cup W \vdash_{P L} \perp$. More generally speaking I will also call imperatives conflicting if they are not obeyable in the given situation.

As prioritized conditional imperatives are our concern here, we let all imperatives in $I$ be ordered by some priority relation $<\subseteq I \times I$. The relation $<$ is assumed to be a strict partial order on $I$, i.e. $<$ is irreflexive and transitive, and additionally we assume $<$ to be well-founded, i.e. infinite descending chains are excluded. For any $i_{1}, i_{2} \in I, i_{1}<i_{2}$ means that $i_{1}$ takes priority over $i_{2}$ (ranks higher than $i_{2}$, is more important than $i_{2}$, etc.). A tuple $\langle I, f, g\rangle$ will be called a conditional imperative structure, and $\langle I, f, g,<\rangle$ a prioritized conditional imperative structure. If all imperatives in $I$ are unconditional, we may drop any reference to the relation $g$ in the tuples and call these basic imperative structures and prioritized imperative structures respectively.

\footnotetext{
${ }^{4}$ Cf. Rescher [35], Sosa [40], van Fraassen [10]. Also cf. Greenspan [12]: "Oughts do not arise, it seems, until it is too late to keep their conditions from being fulfilled."

5 Terms differ here, e.g. Downing [8] uses the term 'compliable' instead of 'obeyable'.
} 


\section{Deontic Concepts}

Given a set of imperatives, one may truly or falsely state that their addressee must, or must not, perform some act or achieve some state of affairs according to what the addressee was ordered to do. For instance, in the 'drinking and driving' example from sec. $1 \mathrm{I}$ think it is true that the agent ought to do the driving, as this is what the second-ranking imperative, uttered by the best friend, requires the agent to do, but that it would be false to say that the agent ought to drink and drive. Statements that something ought to be done or achieved are called 'normative' or 'deontic statements', and the ultimate goal is to find a logical semantics that models the situation and defines the deontic concepts in such a way that the formal results coincide with our natural inclinations in the matter.

\subsection{Deontic operators for unconditional imperatives}

For unconditional imperatives, such definitions are straightforward. Given a basic imperative structure $\mathcal{I}=\langle I, f\rangle$, a monadic deontic $O$-operator is defined by

$(t d-m 1) \quad \mathcal{I} \models O A$ if and only if (iff) $I^{f} \vdash_{P L} A$.

So obligation is defined in terms of what the satisfaction of all imperatives logically implies. With the usual truth definitions for Boolean operators, it can easily be seen that such a definition produces a normal modal operator, i.e. one that is defined by the following axiom schemes plus modus ponens:

(Ext) If $\vdash_{P L} A \leftrightarrow B$, then $O A \leftrightarrow O B$ is a theorem.

(M) $\quad O(A \wedge B) \rightarrow(O A \wedge O B)$

(C) $\quad(O A \wedge O B) \rightarrow O(A \wedge B)$

(N) $O \top$

Furthermore, $(t d-m 1)$ defines standard deontic logic $S D L$, which adds

(D) $\quad O A \rightarrow \neg O \neg A$

iff the imperatives are assumed to be non-conflicting and so $I^{f}$ is $P L$-consistent, i.e. $I^{f} \nvdash_{P L} \perp$. It is immediate that in the case of conflicts, $(t d-m 1)$ pronounces everything as obligatory, and in particular defines $O \perp$ true, thus making the impossible obligatory. If conflicts are not excluded, a solution is to only consider (maximal) subsets of the imperatives whose demands are consistent and define the $O$-operator with respect to these (I write $I \curlywedge \neg C$ for the set of all ' $\neg C$ remainders', i.e. maximal subsets $\Gamma$ of $I$ such that $\left.\Gamma^{f} \nvdash_{P L} \neg C\right)$ :

$(t d-m 2) \quad \mathcal{I} \models O A$ iff $\forall \Gamma \in I \curlywedge \perp: \Gamma^{f} \vdash_{P L} A$

Quite similarly, a dyadic deontic operator $O(A / C)$, meaning that $A$ ought to be true given that $C$ is true, can be defined with respect to the maximal subsets of imperatives that do not conflict in these circumstances:

$(t d-d 1) \quad \mathcal{I} \models O(A / C)$ iff $\forall \Gamma \in I \curlywedge \neg C: \Gamma^{f} \vdash_{P L} A$

So $A$ is obligatory given that $C$ is true if $A$ is what the imperatives in any $\neg C$ remainder demand. In the case of conflicts, this definition produces a "disjunctive solution": e.g. if there are two imperatives ! $A$ and $! B$ with $\vdash_{P L} C \rightarrow(A \rightarrow \neg B)$, then neither $O(A / C)$ nor $O(B / C)$ but $O(A \vee B / C)$ is true. ${ }^{6}$

\footnotetext{
${ }^{6}$ For alternative solutions to the problem of conflicts cf. Goble [11] and my [13], [14].
} 
Often, we want to use the information that we have about the circumstances also for reasoning about the obligations in these circumstances. E.g. if the set of imperatives is $\left\{!\left(p_{1} \vee p_{2}\right)\right\}$, ordering me to either send you a card or phone you, and I cannot send you a card, i.e. $\neg p_{1}$ is true, I should be able to conclude that I should phone you, and so $O\left(p_{2} / \neg p_{1}\right)$ should be true. Such 'circumstantial reasoning' is achieved by the following change to the truth definition:

$(t d-d 2) \quad \mathcal{I} \models O(A / C)$ iff $\forall \Gamma \in I \curlywedge \neg C: \Gamma^{f} \cup\{C\} \vdash_{P L} A$

With the usual truth conditions for Boolean operators, a semantics that employs $(t d-d 2)$ has a sound and (weakly) complete axiom system $P D$ that equals the system $P$ of Kraus, Lehmann, Magidor [23], defined by these axiom schemes

(DExt) If $\vdash_{P L} A \leftrightarrow B$ then $O(A / C) \leftrightarrow O(B / C)$ is a theorem.

$(\mathrm{DM}) \quad O(A \wedge B / C) \rightarrow(O(A / C) \wedge O(B / C))$

$(\mathrm{DC}) \quad O(A / C) \wedge O(B / C) \rightarrow O(A \wedge B / C)$

(DN) $\quad O(\top / C)$

(ExtC) If $\vdash_{P L} C \leftrightarrow D$ then $O(A / C) \leftrightarrow O(A / D)$ is a theorem.

(CCMon) $O(A \wedge D / C) \rightarrow O(A / C \wedge D)$

(CExt) If $\vdash_{P L} C \rightarrow(A \leftrightarrow B)$ then $O(A / C) \leftrightarrow O(B / C)$ is a theorem.

(Or) $\quad O(A / C) \wedge O(A / D) \rightarrow O(A / C \vee D)$

with the additional (restricted) dyadic 'deontic' axiom scheme

(DD-R) If $\nvdash_{P L} \neg C$ then $\vdash_{P D} O(A / C) \rightarrow \neg O(\neg A / C)$

added (hence the name $P D) .^{7}$

\subsection{Deontic operators for conditional imperatives}

Unlike their unconditional counterparts, conditional imperatives have been found hard to reason about. G. H. von Wright [47] called conditional norms the "touchstone of normative logic", and van Fraassen [10] wrote with regard to logics for conditional imperatives: "There may be systematic relations governing this moral dynamics, but I can only profess ignorance of them."

Representing a conditional imperative as an unconditional imperative that demands a material conditional to be made true yields undesired results. Most notorious is the problem of contraposition: consider a set $I$ with the only imperative! $\left(p_{1} \rightarrow p_{2}\right)$, meaning e.g. 'if the police stops you, show your drivers licence'. $(t d-d 1)$ makes true $O\left(p_{2} / p_{1}\right)$, but also $O\left(\neg p_{1} / \neg p_{2}\right)$, so if you can't present your drivers licence (you don't have one) you must see to it that the police does not stop you, which is hardly what the speaker meant you to do.One may think that such problems arise from the fact that antecedents of conditional imperatives often describe states of the affairs that the agent is not supposed to, and often cannot, control. But consider the set $\left\{!\left(p_{1} \rightarrow p_{2}\right), !\left(\neg p_{1} \rightarrow p_{3}\right)\right\}$, it yields $O\left(p_{2} / \neg p_{3}\right)$ with $(t d-d 1)$. Here, $p_{2}$ is what the consequent of some imperative demands, so it supposedly describes something the agent can control. Now let

\footnotetext{
${ }^{7}$ For proofs, and an additional "credulous ought" that defines $O(A / C)$ true if the truth of $A$ is required to satisfy all imperatives in some $\neg C$-remainder, cf. my [14].
} 
the imperatives be interpreted as ordering me to wear my best suit if it does not rain, and a rain coat if it does: it is clear nonsense that I am obliged to wear a raincoat given that I can't wear my best suit (e.g. it is in the laundry). Such problems are the reason why we cautiously use special models for conditional imperatives (i.e. conditional imperative structures), and write $p_{1} \Rightarrow ! p_{2}$ instead of $!\left(p_{1} \rightarrow p_{2}\right)$. But this only delegates the problem from the level of representation to that of semantics, where now new truth definitions must be found.

Let $\mathcal{I}=\langle I, f, g\rangle$ be a conditional imperative structure, and let us ignore for the moment the further complication of possible conflicts between imperatives. Then the following seems a natural way to define what ought to be the case in circumstances where $C$ is assumed to be true:

$(t d-c d 1) \quad \mathcal{I} \models O(A / C)$ iff $\left[\text { Triggered }_{\mathcal{I}}(\{C\}, I)\right]^{f} \vdash_{P L} A$

So dyadic obligation is defined in terms what is necessary to satisfy all imperatives that are triggered in the assumed circumstances. E.g. if $I=\left\{p_{1} \Rightarrow ! p_{2}\right\}$, with its only imperative interpreted as "if you have a cold, stay in bed", then $O\left(p_{2} / p_{1}\right)$ truly states that I must stay in bed given that I have a cold.

Like in the unconditional case, it seems important to be able to use 'circumstantial reasoning', i.e. employ the information about the situation not only to determine if an imperative is triggered, but also for reasoning with its consequent. E.g. if the set of imperatives is $\left\{p_{1} \Rightarrow !\left(p_{2} \vee p_{3}\right)\right\}$, with its imperative interpreted as expressing "if you have a cold, either stay in bed or wear a scarf", one would like to obtain $O\left(p_{3} / p_{1} \wedge \neg p_{2}\right)$, expressing that given that $I$ have a cold and don't stay in bed, I must wear a scarf. So $(t d-c d 1)$ may be changed into

$\left(t d\right.$-cd2) $\mathcal{I} \models O(A / C)$ iff $\left[\text { Triggered }_{\mathcal{I}}(\{C\}, I)\right]^{f} \cup\{C\} \vdash_{P L} A$.

Though the step from $(t d-c d 1)$ to $(t d-c d 2)$ seems quite reasonable, such definitions have also been criticized for defining the assumed circumstances as obligatory. E.g. if the set of imperatives is $\left\{p_{1} \Rightarrow ! p_{2}\right\}$, where the imperative is interpreted as expressing "if you hit someone, apologize to him", then (td-5) makes true $O\left(p_{1} \wedge p_{2} / p_{1}\right)$, and hence also $O\left(p_{1} / p_{1}\right)$, so given that I hit someone, this is something I ought to do. The criticism looses much of its edge in the present setting, where one can point to the distinction between imperatives (there is no imperative that demands $p_{1}$ ) and ought sentences that describe what must be true when all triggered imperatives are satisfied in the supposed circumstances: then the truth of $O\left(p_{1} / p_{1}\right)$ seems no more paradoxical than the truth of $O \top$ that is accepted in most systems of deontic logic.

\subsection{Further modifications}

In Makinson \& van der Torre's [25] more general theory of 'input/output logic', $(t d-c d 1)$ is termed 'simple-minded output', and $(t d-c d 2)$ is its 'throughput version'. ${ }^{8}$ As the names suggests, the authors also discuss more refined operations, which again might be considered for reasoning about conditional imperatives. One modification addresses the possibility of 'reasoning by cases' that e.g. makes

\footnotetext{
${ }^{8}$ If $I$ resembles the generating set $G$ of input/output logic, then $O(A / C)$ means that
} $A$ is an output given the input $C$ (Makinson \& van der Torre write $A \in \operatorname{out}(G,\{C\})$ ). 
true $O\left(p_{2} \vee p_{4} / p_{1} \vee p_{3}\right)$ for a set of imperatives $I=\left\{p_{1} \Rightarrow ! p_{2}, p_{3} \Rightarrow ! p_{4}\right\}$. This may be achieved by the following definition, where $\mathscr{L}_{P L} \perp \neg C$ is the set of all maximal subsets of the language $\mathscr{L}_{P L}$ that are consistent with $C:{ }^{9}$

$\left(t d\right.$-cd3) $\quad \mathcal{I} \models O(A / C) \quad$ iff $\forall V \in \mathscr{L}_{P L} \perp \neg C:\left[\operatorname{Triggered}_{\mathcal{I}}(V, I)\right]^{f} \vdash_{P L} A$

In the example, each set $V \subset \mathscr{L}_{P L}$ that is maximally consistent with $p_{1} \vee p_{3}$ either contains $p_{1}$, then $p_{1} \Rightarrow ! p_{2}$ is triggered and so $p_{2}$ and also $p_{2} \vee p_{4}$ is implied by $\left[\operatorname{Triggered}_{\mathcal{I}}(V, I)\right]^{f}$, or it contains $\neg p_{1}$, but then it cannot also contain $\neg p_{3}$ and so must contain $p_{3}$, so $p_{3} \Rightarrow ! p_{4}$ is triggered and therefore $p_{4}$ and also $p_{2} \vee p_{4}$ implied, so for all sets $V, p_{2} \vee p_{4}$ is implied and so $O\left(p_{2} \vee p_{4} / p_{1} \vee p_{3}\right)$ made true.

In order to add 'circumstantial reasoning' to $(t d-c d 3)$ - or, in Makinson \& van der Torre's terms, for its 'throughput version' -, one might, in the vein of $(t d-d 2)$ and $(t d-c d 2)$, try this definition:

$\left(t d-c d 4^{-}\right) \mathcal{I} \models O(A / C)$ iff $\forall V \in \mathscr{L}_{P L} \perp \neg C:\left[\operatorname{Triggered}_{\mathcal{I}}(V, I)\right]^{f} \cup\{C\} \vdash_{P L} A$

But the definition seems too weak. Consider the set $\left\{p_{1} \Rightarrow !\left(\neg p_{2} \vee p_{4}\right), p_{3} \Rightarrow ! p_{4}\right\}$ and the situation $\left(p_{1} \wedge p_{2}\right) \vee p_{3}$. We would expect a reasoning as follows: in this situation, either $p_{1} \wedge p_{2}$ is true, so the first imperative is triggered but we cannot satisfy it by bringing about $\neg p_{2}$, and so must bring about $p_{4}$. Or $p_{3}$ is true, then the second imperative is triggered and we must again bring about $p_{4}$. So we must bring about $p_{4}$ in the given situation. But the definition fails to make true $O\left(p_{4} /\left(p_{1} \wedge p_{2}\right) \vee p_{3}\right)$. Like Makinson and van der Torre [25], I therefore combine reasoning by cases with a stronger version of throughput:

$\left(t d\right.$-cd4) $\quad \mathcal{I} \models O(A / C) \quad$ iff $\forall V \in \mathscr{L}_{P L} \perp \neg C:\left[\operatorname{Triggered}_{\mathcal{I}}(V, I)\right]^{f} \cup V \vdash_{P L} A$

As is easy to see, this resolves the difficulty: for $\left\{p_{1} \Rightarrow !\left(\neg p_{2} \vee p_{4}\right), p_{3} \Rightarrow ! p_{4}\right\}$, $O\left(p_{4} /\left(p_{1} \wedge p_{2}\right) \vee p_{3}\right)$ is now true, as desired. However, this modification has a surprising consequence: it makes the reasoning about conditional imperatives collapse into reasoning about consequences of their materializations:

Observation $1 B y(t d-c d 4), \mathcal{I}=O(A / C)$ iff $m(I) \cup\{C\} \vdash_{P L} A$.

Proof. For the right-to-left direction, for any imperative $i \in I$ and any set $V \in \mathscr{L}_{P L} \perp \neg C$, either $V$ includes $g(i)$, so $i \in \operatorname{Triggered}_{\mathcal{I}}(V, I)$ and therefore $\left[\operatorname{Triggered}_{\mathcal{I}}(V, I)\right]^{f} \vdash_{P L} g(i) \rightarrow f(i)$, or it does not include $g(i)$, but then it includes $\neg g(i)$ by maximality, hence $V \vdash_{P L} g(i) \rightarrow f(i)$. So $\left[\operatorname{Triggered}_{\mathcal{I}}(V, I)\right]^{f} \cup$ $V \vdash_{P L} g(i) \rightarrow f(i)$. For the left-to-right direction, if $m(I) \cup\{C\} \nvdash_{P L} A$ then $m(I) \cup\{C\} \cup\{\neg A\}$ is consistent, so there is a $V \in \mathscr{L}_{P L} \perp \neg C$ such that $m(I) \cup\{C\} \cup$ $\{\neg A\} \subseteq V$. It is immediate that for each $i \in \operatorname{Triggered}_{\mathcal{I}}(V, I), m(I) \cup V \vdash_{P L} f(i)$, so if $\left[\operatorname{Triggered}_{\mathcal{I}}(V, I)\right]^{f} \cup V \vdash_{P L} A$ then $m(I) \cup V \vdash_{P L} A$ and since $m(I) \subseteq V$ also $V \vdash_{P L} A$. Since $V$ was consistent and included $\neg A$, it cannot also derive $A$, and so by contraposition $\left[\operatorname{Triggered}_{\mathcal{I}}(V, I)\right]^{f} \cup V \nvdash_{P L} A$.

But such an equivalence makes all the problems discussed above for identifying conditional imperatives with unconditional imperatives that demand their mate-

\footnotetext{
${ }^{9}$ Makinson \& van der Torre's [25] call the resulting operator 'basic output', of which a syntactical version was first presented by Świrydowicz [41] p. 32.
} 
rializations reappear, in particular the problem of contraposition. ${ }^{10}$ So it seems we must choose between 'reasoning by cases' and 'circumstantial reasoning'. ${ }^{11}$

Another modification that these authors consider is that of 'reusable output': when an imperative is triggered that demands $A$, and $A$ is the trigger for some imperative $A \Rightarrow ! B$, then we also ought to do $B$. Such a modification can easily be incorporated into a truth definition and its 'throughput' version:

$(t d-c d 5) \quad \mathcal{I} \models O(A / C)$ iff $\left[\operatorname{Triggered}_{\mathcal{I}}^{*}(\{C\}, I)\right]^{f} \vdash_{P L} A$

$\left(t d\right.$-cd6) $\mathcal{I} \models O(A / C)$ iff $\left[\text { Triggered }_{\mathcal{I}}^{*}(\{C\}, I)\right]^{f} \cup\{C\} \vdash_{P L} A$

where $\operatorname{Triggered}_{\mathcal{I}}^{*}(W, \Gamma)$ means the smallest subset of $\Gamma \subseteq I$ such that for all $i \in$ $\Gamma$, if $\left[\operatorname{Triggered}_{\mathcal{I}}^{*}(W, \Gamma)\right]^{f} \cup W \vdash_{P L} g(i)$ then $i \in \operatorname{Triggered}_{\mathcal{I}}^{*}(W, \Gamma)$. Moreover, the two modifications of 'reasoning by cases' and 'reusable output' can be combined to produce the following definition and its 'throughput' variant:

$(t d-c d 7) \quad \mathcal{I} \models O(A / C)$ iff $\forall V \in \mathscr{L}_{P L} \perp \neg C:\left[\text { Triggered }_{\mathcal{I}}^{*}(V, I)\right]^{f} \vdash_{P L} A$

$\left(t d\right.$-cd8) $\mathcal{I} \models O(A / C)$ iff $\forall V \in \mathscr{L}_{P L} \perp \neg C:\left[\text { Triggered }_{\mathcal{I}}^{*}(V, I)\right]^{f} \cup V \vdash_{P L} A$

The topic of 'reusable output' is discussed under the name of 'deontic detachment' in the literature on deontic logic, and there is no agreement whether such a procedure is admissible (Makinson [24] p. 43 argues in favor, whereas Sven Ove Hansson [17] p. 155 disagrees). E.g. let $I=\left\{! p_{1}, p_{1} \Rightarrow ! p_{2}, \neg p_{1} \Rightarrow ! \neg p_{2}\right\}$, and for its interpretation assume that it is imperative for the proper execution of your job that you develop novel methods, which make you eligible for a bonus, and that if you develop such novel methods you owe it to yourself to apply for the bonus, but that if you don't develop such methods you must not apply for the bonus. Truth definitions that accept 'deontic detachment' make true $O\left(p_{2} / \top\right)$, and so tell us that you ought to apply for the bonus, which seems weird since it may be that you never invent anything. However, proponents of deontic detachment may argue that in such a situation, $O\left(p_{1} \wedge p_{2} / \top\right)$ should hold, i.e. you ought to invent new methods and apply for the bonus, and that the reluctance to also accept $O\left(p_{2} / \top\right)$ is - like the inference from "you ought to put on your parachute and jump" to "you ought to jump" - just a variant of Ross' Paradox that is usually considered harmless.

For $(t d-c d 7)$ we once again obtain $O\left(p_{2} / \neg p_{3}\right)$ for $I=\left\{p_{1} \Rightarrow ! p_{2}, \neg p_{1} \Rightarrow ! p_{3}\right\}$ : for any $V \in \mathscr{L}_{P L} \perp p_{3}, \neg p_{3} \in V$, furthermore either $p_{1} \in V$ and so $p_{1} \Rightarrow ! p_{2} \in$ $\operatorname{Triggered}_{\mathcal{I}}^{*}(V, I)$, or $\neg p_{1} \in V$, then $\neg p_{1} \Rightarrow$ ! $p_{3} \in \operatorname{Triggered}_{\mathcal{I}}^{*}(V, I)$, and since $\left\{p_{3}\right\} \cup$

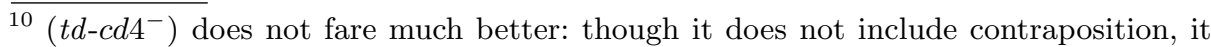
again makes $O\left(p_{2} / \neg p_{3}\right)$ true for $I=\left\{p_{1} \Rightarrow ! p_{2}, \neg p_{1} \Rightarrow ! p_{3}\right\}$, which is counterintuitive.

${ }^{11}$ Legal use of 'reasoning by cases', or Wahlfeststellung, is controversial. It means that if the defendant either committed crime $\alpha$ or crime $\beta$, the defendant would be convicted according to the milder law. A proponent would argue that since the defendant committed a crime (though it remains open which), justice demands that he should not go free, while the defense would argue that this violates the in dubio pro reo principle, since neither charge is sufficiently proved. After a Reichsgericht ruling in 1934 allowed Wahlfeststellung for cases in which the crimes in question were 'ethically and psychologically equivalent', the national-socialist lawmakers introduced a law prescribing its unrestricted application in 1935, considered ideological and lifted again by the Allied Control Council of Germany in 1946 (cf. [43]). 
$\left\{\neg p_{3}\right\} \vdash_{P L} p_{1}$, again $p_{1} \Rightarrow ! p_{2}$ is in Triggered $_{\mathcal{I}}^{*}(V, I)$, hence $\left[\text { Triggered }_{\mathcal{I}}^{*}(V, I)\right]^{f} \vdash_{P L}$ $p_{2}$ for all $V \in \mathscr{L}_{P L} \perp p_{3}$. But as we saw above, when interpreting the imperatives as 'if it rains, wear a raincoat' and 'if it does not rain, wear your best jacket', this result seems counterintuitive. ${ }^{12}$ Note that $(t d-c d 8)$ is again equivalent to $\mathcal{I} \models O(A / C)$ iff $m(I) \cup\{C\} \vdash_{P L} A$ and thus to ( $t d-c d 4$ ) (cf. Makinson \& van der Torre [25] observation 16; [26], p. 156):

Observation $2 B y(t d-c d 8), \mathcal{I} \models O(A / C)$ iff $m(I) \cup\{C\} \vdash_{P L} A$.

Proof. Similar to the proof of observation 1. For the left-to-right direction, use that for each $i \in \operatorname{Triggered}_{\mathcal{I}}^{*}(V, I), m(I) \cup V \vdash_{P L} f(i)$, which is immediate.

\subsection{Operators for prioritized conditional imperatives}

This paper focuses on prioritized conditional imperatives, and for these there is a further hurdle to finding the proper truth definitions for deontic concepts. Priorities are only required if the imperatives cannot all be obeyed - otherwise there is no reason not to obey all, and the priority ordering is not used. So the truth definitions must be able to deliver meaningful results for possibly conflicting imperatives. The intuitive idea is to use the information in the ordering to choose subsets of the set of imperatives under consideration that contain only the more important imperatives and leave out less important, conflicting ones, so that the resulting 'preferred subset' (or rather, subsets, since the choice may not always be determined by the ordering) only contains imperatives that do not conflict in the given situation. More generally, let $\mathcal{I}$ be a prioritized conditional imperative structure $\langle I, g, f,<\rangle$, and let $\Delta$ be a subset of $I$. Then $\mathscr{P}_{\mathcal{I}}(W, \Delta)$ contains just the subsets of $\Delta$ that are thus preferred given the world facts $W$. The above truth definitions can then be adapted such that they now describe something as obligatory iff it is so with respect to all the preferred subsets of the imperatives, i.e. they take on the following forms:

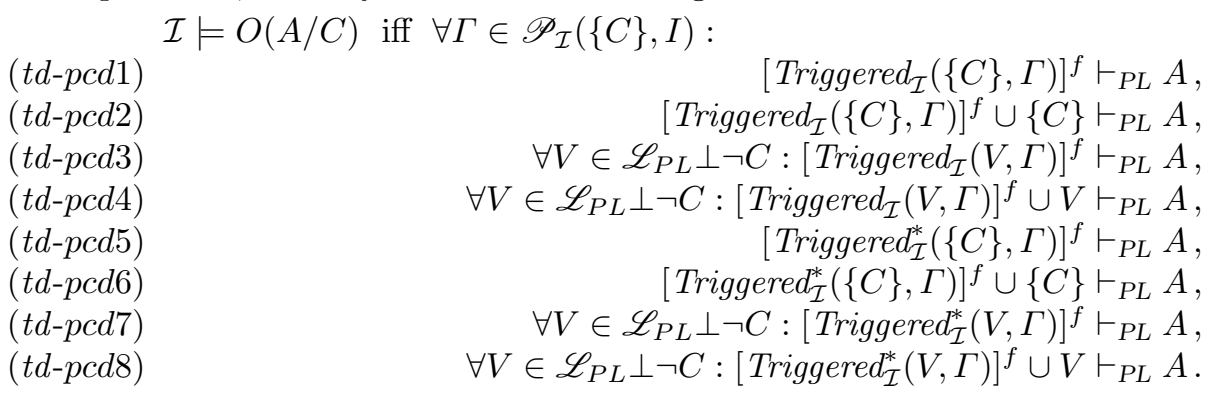

So e.g. ( $t d-p c d 1)$ defines $A$ as obligatory if the truth of $A$ is required to satisfy the triggered imperatives in any preferred subset. Of course, the crucial and as yet missing element is the decision procedure that determines the set $\mathscr{P}_{\mathcal{I}}(\{C\}, I)$ of preferred subsets. The next section discusses several proposals to define such subsets; a new proposal is presented in the section that follows it.

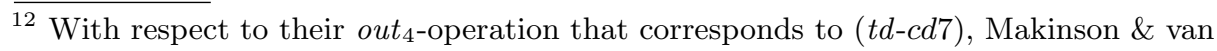
der Torre [25] speak of a 'ghostly contraposition'. 


\section{Identifying the Preferred Subsets}

\subsection{Brewka's preferred subtheories}

The idea that normative conflicts can be overcome by use of a priority ordering of the norms involved dates back at least to Ross [37] and is also most prominent in von Wright's work (cf. [45] p. 68, 80). However, it has turned out to be difficult to determine the exact mechanism by which such a resolution of conflicts can be achieved. This is true even when only unconditional imperatives are considered, and when special problems are left out of the picture, such that the ordering itself might be dependent on the facts (e.g. when the command of an officer in the field may override that of her superior due to unexpected circumstances), or be the subject of normative regulation (e.g. when we are commanded to obey the law of God more than the law of man). Discussing various proposals for resolution of conflicts between unconditional imperative, I have argued in [15] that an 'incremental' definition be used for determining the relevant subsets. Based on earlier methods by Rescher [34], such a definition was first introduced by Brewka [4] for reasoning with prioritized defaults. For any priority relation $<$, the idea is to consider all the 'full prioritizations' $\prec$ of $<$ (strict well orders that preserve $<$ ), and then work ones way from top to bottom by adding the $\prec$-nexthigher imperative to the thus constructed 'preferred subtheory' if its demand is consistent with the given facts and the demands of the imperatives that were added before. For the present setting, the definition can be given as follows:

Definition 1 (Brewka's preferred subtheories).

Let $\mathcal{I}=\langle I, f, g,<\rangle$ be a prioritized conditional imperative structure, $\Delta$ be a subset of $I$, and $W \subseteq \mathscr{L}_{P L}$ be a set of PL-sentences. Then $\Gamma \in \mathscr{P}_{\mathcal{I}}^{\mathrm{B}}(W, \Delta)$ iff (i) $W \nvdash_{P L} \perp$, and (ii) $\Gamma$ is obtained from a full prioritization $\prec$ by defining

$\Gamma_{[\prec \downarrow i]}= \begin{cases}\bigcup_{j \prec i} \Gamma_{[\prec \downarrow j]} \cup\{i\} & \text { if } W \cup\left[\bigcup_{j \prec i} \Gamma_{[\prec \downarrow j]} \cup\{i\}\right]^{f} \nvdash_{P L} \perp, \text { and } \\ \bigcup_{j \prec i} \Gamma_{[\prec \downarrow j]} & \text { otherwise, }\end{cases}$

for any $i \in \Delta$, and letting $\Gamma=\bigcup_{i \in \Delta} \Gamma_{[\prec \downarrow i]}$.

Clause (i) ensures that for an inconsistent set of assumed 'facts', no set is preferred. Somewhat roundabout, owed to the possibility of infinite ascending subchains, clause (ii) then recursively defines a set $\Gamma \in \mathscr{P}_{\mathcal{I}}^{\mathrm{B}}(W, \Delta)$ for each full prioritization $\prec$ : take the $\prec$-first $i$ (the exclusion of infinite descending subchains guarantees that it exists) and if $W \cup\left\{i^{f}\right\} \nvdash_{P L} \perp$ then let $\Gamma_{[\prec \downarrow i]}=\{i\}$; otherwise $\Gamma_{[\prec \downarrow i]}$ is left empty. ${ }^{13}$ Similarly, any $\prec$-later $i$ is tested for possible addition to the set $\bigcup_{j \prec i} \Gamma_{[\prec \downarrow j]}$ of elements that were added in the step for a $j \in \Delta$ that occurs $\prec$-prior to $i$. $\Gamma$ is then the union of all these sets.

To see how this definition works, consider the set $I=\left\{!\left(p_{1} \vee p_{2}\right), ! \neg p_{2}, ! \neg p_{1}\right\}$, with the ranking ! $\left(p_{1} \vee p_{2}\right)<! \neg p_{1}$ and $! \neg p_{2}<! \neg p_{1}$. For an interpretation, let $\left.!\left(p_{1} \vee p_{2}\right)\right)$ be your mother's request that you buy cucumbers or spinach for dinner, ! $\neg p_{1}$ be your father's wish that no cucumbers are bought, and $! \neg p_{2}$ your

\footnotetext{
${ }^{13}$ As usual, the union of an empty set of sets is taken to be the empty set.
} 
sister's desire that you don't buy any spinach. We have two full prioritizations $!\left(p_{1} \vee p_{2}\right)<! \neg p_{2}<! \neg p_{1}$ and $! \neg p_{2}<!\left(p_{1} \vee p_{2}\right)<! \neg p_{1}-$ let these be termed $\prec_{1}$ and $\prec_{2}$, respectively. The construction for $\prec_{1}$ adds the imperative ! $\left(p_{1} \vee p_{2}\right)$ in the first step and, since no conflict with the situation arises, $! \neg p_{2}$ in the second step. In the third and last step, nothing is added since $! \neg p_{1}$ conflicts with the demands of the already added imperatives. For $\prec_{2}$ the only difference is that the first two imperatives are added in inverse order. Thus $\mathscr{P}_{\mathcal{I}}^{\mathrm{B}}(W, I)=\left\{\left\{!\left(p_{1} \vee p_{2}\right), ! \neg p_{2}\right\}\right\}$. Using ( $t d-p c d 2$ ) we obtain $O\left(p_{1} \wedge \neg p_{2} / \top\right.$ ), which means that you have to buy spinach and not cucumbers, thus fulfilling your parents' requests but not your sister's, which seems reasonable.

As I showed in [15], Brewka's method is extremely successful for dealing with unconditional imperatives. It is provably equivalent for such imperatives to methods proposed by Ryan [38] and Sakama \& Inoue [39], and it avoids problems of other approaches by Alchourrón \& Makinson [2], Prakken [31] and Prakken \& Sartor [32]. Moreover, an equally intuitive maximization method proposed by Nebel [29], [30], that adds first a maximal number of the highest-ranking imperatives, then a maximal number of the second-ranking imperatives, etc., but for its construction requires the ordering to be based on a complete preorder, can be shown to be embedded in Brewka's approach for such orderings. So my aim will be to retain Brewka's method for the unconditional case. However, when it is applied without change to conditional imperatives, the algorithm may lead to incorrect results. E.g. consider a set $I$ with two equally ranking imperatives $\left\{p_{1} \Rightarrow\right.$ $\left.! p_{2}, \neg p_{1} \Rightarrow ! \neg p_{2}\right\}$, meaning e.g. "if you go out, wear your boots" and "if you don't go out, don't wear your boots". Since the consequents contradict each other, an unmodified application of Brewka's method produces $\mathscr{P}_{\mathcal{I}}^{\mathrm{B}}\left(\left\{p_{1}\right\}, I\right)=\left\{\left\{p_{1} \Rightarrow\right.\right.$ $\left.\left.! p_{2}\right\},\left\{\neg p_{1} \Rightarrow ! \neg p_{2}\right\}\right\}$, which fails to make true $O\left(p_{2} / p_{1}\right)$ by any truth definition ( $t d-p c d 1-8)$ : the right set contains no imperatives that are in any way triggered by $p_{1}$. So we cannot derive that you ought to wear your boots, given that you are going out. But intuitively there is no conflict, since the conflicting obligations arise in mutually exclusive circumstances only.

\subsection{A naïve approach}

A straightforward way to adopt Brewka's method to the case of conditional imperatives is to use not all imperatives for the construction, but only those that are triggered by the facts $W$, i.e. to use $\operatorname{Triggered}_{\mathcal{I}}(W, \Delta)$ instead of $\Delta$ :

Definition 2 (The naïve approach).

Let $\mathcal{I}=\langle I, f, g,<\rangle$ be a prioritized conditional imperative structure, $\Delta$ be a subset of $I$, and $W \subseteq \mathscr{L}_{P L}$ be a set of PL-sentences. Then $\Gamma \in \mathscr{P}_{\mathcal{I}}(W, \Delta)$ iff $\Gamma \in \mathscr{P}_{\mathcal{I}}^{\mathrm{B}}\left(W\right.$, Triggered $\left._{\mathcal{I}}(W, \Delta)\right)$.

The change resolves our earlier problems with Brewka's method: consider again the set of imperatives $\left\{p_{1} \Rightarrow ! p_{2}, \neg p_{1} \Rightarrow ! \neg p_{2}\right\}$, where the imperatives were interpreted as ordering me to wear my boots when I go out, and not wear my boots when I don't. The new definition produces $\mathscr{P}_{\mathcal{I}}^{\mathrm{n}}\left(\left\{p_{1}\right\}, I\right)=\left\{\left\{p_{1} \Rightarrow ! p_{2}\right\}\right\}$, its only 
'preferred' subset containing just the one imperative that is triggered given the facts $\left\{p_{1}\right\}$. By any truth definition ( $\left.t d-p c d 1-8\right), O\left(p_{2} / p_{1}\right)$ is now defined true, so given that you go out, you ought to wear your boots, which is as it should be.

The naïve approach is clearly a conservative extension of Brewka's original method to conditional imperatives: for sets $\Delta$ of unconditional imperatives, Triggered $_{\mathcal{I}}(\{\top\}, \Delta)=\Delta$. It is similar to Horty's proposal in [21] in that conflicts are only removed between imperatives that are triggered (though the exact mechanism differs from Horty's). When I nevertheless call it 'naïve', this is because there are conceivable counterexamples to this method. Consider the set of imperatives $\left\{! p_{1}, p_{1} \Rightarrow ! p_{2}, ! \neg p_{2}\right\}$, ranked $! p_{1}<p_{1} \Rightarrow ! p_{2}<! \neg p_{2}$, and for an interpretation suppose that your job requires you to go outside $p_{1}$, that your mother, who is concerned for your health, told you to wear a scarf $p_{2}$ if you go outside, and that your friends don't want you to wear a scarf, whether you go outside or not. In the default situation $T$ only the first imperative and the third are triggered, i.e. Triggered ${ }_{\mathcal{I}}(\{\top\}, I)=\left\{! p_{1}, ! \neg p_{2}\right\}$. Since their demands are consistent with each other, we obtain $\mathscr{P}_{\mathcal{I}}^{\mathrm{n}}(\{\top\}, I)=\left\{\left\{! p_{1}, ! \neg p_{2}\right\}\right\}$, for which all truth definitions ( $t d-p c d 1-8)$ make $O\left(p_{1} \wedge \neg p_{2} / T\right)$ true. So you ought to go out and not wear a scarf, thus satisfying the first and the third imperative, but violating the second-ranking imperative. But arguably, if an imperative is to be violated, it should not be the second-ranking $p_{1} \Rightarrow ! p_{2}$, but the lowest ranking $! \neg p_{2}$ instead.

\subsection{The stepwise approach}

To avoid the difficulties of the 'naïve' approach, it seems we must not just take into account the imperatives that are triggered, but also those that become triggered when higher ranking imperatives are satisfied. To this effect, the following modification may seem reasonable:

Definition 3 (The stepwise approach).

Let $\mathcal{I}=\langle I, f, g,<\rangle$ be a prioritized conditional imperative structure, $\Delta$ be a subset of $I$, and $W \subseteq \mathscr{L}_{P L}$ be a set of PL-sentences. Then $\Gamma \in \mathscr{P}^{\mathrm{s}}(W, \Delta)$ iff (i) $W \nvdash_{P L} \perp$, and (ii) $\Gamma$ is obtained from a full prioritization $\prec$ by defining

$\Gamma_{[\prec \downarrow i]}= \begin{cases}\bigcup_{j \prec i} \Gamma_{[\prec \downarrow j]} \cup\{i\} & \text { if } i \in \text { Satisfiable }_{\mathcal{I}}\left(W \cup\left[\bigcup_{j \prec i} \Gamma_{[\prec \downarrow j]}\right]^{f}, \Delta\right), \text { and } \\ \bigcup_{j \prec i} \Gamma_{[\prec \downarrow j]} & \text { otherwise, }\end{cases}$ for any $i \in \Delta$, and letting $\Gamma=\bigcup_{i \in \Delta} \Gamma_{[\prec \downarrow i]}$.

So at each step one considers what happens if the imperatives that were included so far are satisfied, and adds the current imperative only if it is satisfiable given the truth of $W$ and the satisfaction of these previous imperatives. Note that satisfiability of an imperative, like its satisfaction and violation, presupposes that the imperative is triggered. In contrast to the naïve approach, the new definition not only includes, at each step, those imperatives that are triggered and can be satisfied given the facts and the supposed satisfaction of the previously added imperatives: it also includes those that become triggered when a previously added imperative is satisfied. 
The modification avoids the previous difficulty: consider again the set of imperatives $\left\{! p_{1}, p_{1} \Rightarrow ! p_{2}, ! \neg p_{2}\right\}$, with the ranking $! p_{1}<p_{1} \Rightarrow ! p_{2}<! \neg p_{2}$. There is just one full prioritization, which for $W=\{\top\}$ yields in the first step the set $\left\{! p_{1}\right\}$, and in the second step $\left\{! p_{1}, p_{1} \Rightarrow ! p_{2}\right\}$, since $p_{1} \Rightarrow ! p_{2}$ is triggered when the previously added imperative ! $p_{1}$ is assumed to be satisfied. In the third step, nothing is added: though the imperative $! \neg p_{2}$ is triggered, it cannot be satisfied together with the previously added imperatives. So we obtain $\mathscr{P}_{\mathcal{I}}^{\mathrm{s}}(\{\top\}, I)=$ $\left\{\left\{! p_{1}, p_{1} \Rightarrow ! p_{2}\right\}\right\}$, and hence $O\left(p_{1} / \top\right)$, but not $O\left(p_{1} \wedge \neg ! p_{2} / \top\right)$, is defined true by all of ( $t d-p c d 1-8)$. Operators that accept 'deontic detachment' (as defined by $t d$-pcd5-8) even make true $O\left(p_{1} \wedge p_{2} / \top\right)$, and so in the given interpretation you must go out and wear a scarf, which now is as it should be.

However, a small change in the ordering shows that this definition does not suffice: let the imperatives now be ranked $p_{1} \Rightarrow ! p_{2}<! p_{1}<! \neg p_{2}$. (For the interpretation, assume that the conditional imperative to wear a scarf when leaving the house was uttered by some high-ranking authority, e.g. a doctor.) Then again $\mathscr{P}_{\mathcal{I}}^{\mathrm{s}}(\{\top\}, I)=\left\{\left\{! p_{1}, ! \neg p_{2}\right\}\right\}$ : in the first step, nothing is added since $p_{1} \Rightarrow ! p_{2}$ is neither triggered by the facts nor by the assumed satisfaction of previously added imperatives (there are none). In the next two steps, ! $p_{1}$ and $! \neg p_{2}$ are added, as each is consistent with the facts and the satisfaction of the previously added imperatives. So again all of ( $t d-p c d 1-8)$ make true $O\left(p_{1} \wedge \neg p_{2} / T\right)$, i.e. you ought to go out and not wear a scarf, satisfying the second and third ranking imperatives at the expense of the highest ranking one. But surely, if one must violate an imperative, it should be one of the lower-ranking ones instead.

\subsection{The reconsidering approach}

The merits of the stepwise approach were that it did not only consider the imperatives that are triggered, but also those that become triggered when already added imperatives are satisfied. Such considerations applied to those imperatives that follow in the procedure. Yet the satisfaction of already added imperatives might also trigger higher-ranking imperatives, which by this method are not considered again. So it seems necessary, at each step, to reconsider also the higher-ranking imperatives. An algorithm that does that was first introduced for default theory by Marek \& Truszczyński [28] p. 72, and later employed by Brewka in [5]; it can be reformulated for the present setting as follows:

Definition 4 (The reconsidering approach).

Let $\mathcal{I}=\langle I, f, g,<\rangle$ be a prioritized conditional imperative structure, $\Delta$ be a subset of $I$, and $W \subseteq \mathscr{L}_{P L}$ be a set of PL-sentences. Then $\Gamma \in \mathscr{P}_{\mathcal{I}}^{\mathrm{r}}(W, \Delta)$ iff (i) $W \nvdash_{P L} \perp$, and (ii) $\Gamma$ is obtained from a full prioritization $\prec$ by defining

$$
\Gamma_{i}=\bigcup_{j \prec i} \Gamma_{\beta} \cup \min _{\prec}\left[\text { Satisfiable }_{\mathcal{I}}\left(W \cup\left[\bigcup_{j \prec i} \Gamma_{j}\right]^{f}, \Delta\right) \backslash \bigcup_{j \prec i} \Gamma_{j}\right]
$$

for $i \in \Delta$, and letting $\Gamma=\bigcup_{i \in \Delta} \Gamma_{i}$.

The definition reconsiders at each step the whole ordering, and adds the $\prec$-first ${ }^{14}$ imperative that has not been added previously and is satisfiable given both the

${ }^{14}$ For any ordering $<$ on some set $\Gamma, \min _{<} \Gamma=\left\{i \in \Gamma \mid \forall i^{\prime} \in \Gamma:\right.$ if $i^{\prime} \neq i$, then $\left.i^{\prime} \nless i\right\}$, and $\max _{<} \Gamma=\left\{i \in \Gamma \mid \forall i^{\prime} \in \Gamma:\right.$ if $i^{\prime} \neq i$, then $\left.i \nless i^{\prime}\right\}$, as usual. 
circumstances $C$ and the consequents of the previously added imperatives. Note that in ' $\Gamma_{i}$ ', $i$ is used just as a index - it does not mean that $i$ is considered for addition to the set at this step, and in fact it may be added at an earlier or later step (or not at all). To see how the definition works, consider again the example which the stepwise approach failed, i.e. the set of imperatives $\left\{! p_{1}, p_{1} \Rightarrow\right.$ $\left.! p_{2}, ! \neg p_{2}\right\}$, with the ranking $p_{1} \Rightarrow ! p_{2}<! p_{1}<! \neg p_{2}$. We are interested in the preferred sets for the default circumstances $\top$, i.e. the sets in $\mathscr{P}_{\mathcal{I}}^{\mathrm{r}}(\{\top\}, I) . I$ is already fully prioritized, so there is just one such set. Applying the algorithm, we find the minimal (highest ranking) element in Satisfiable $_{\mathcal{I}}(\{\top\}, I)$ is ! $p_{1}$, so this element is added in the first step. In the second step, we look for the minimal element in Satisfiable $_{\mathcal{I}}\left(\{\top\} \cup\left\{! p_{1}\right\}^{f}, I\right)$, other than the previously added ! $p_{1}$. It is $p_{1} \Rightarrow ! p_{2}$, since the assumed satisfaction of all previously added imperatives triggers it, and its consequent can be true together with $\{\top\} \cup\left\{p_{1}\right\}$. So $p_{1} \Rightarrow$ $! p_{2}$ is added in this step. In the remaining third step, nothing is added: ! $\neg p_{2}$ is not in Satisfiable $_{\mathcal{I}}\left(\{\top\} \cup\left\{! p_{1}, p_{1} \Rightarrow ! p_{2}\right\}^{f}, I\right)$, and all other elements in this set have been previously added. So $\mathscr{P}_{\mathcal{I}}^{\mathrm{r}}(\{\top\}, I)=\left\{\left\{! p_{1}, p_{1} \Rightarrow ! p_{2}\right\}\right\}$. Now all truth definitions ( $t d-p c d 1-8)$ make true $O\left(p_{1} / \top\right)$, but not $O\left(p_{1} \wedge \neg ! p_{2} / \top\right)$, and operators that accept 'deontic detachment' make true $O\left(p_{1} \wedge p_{2} / \top\right)$. So, in the given interpretation, you must go out (as your job requires) and wear a scarf (as the doctor ordered you to do in case you go out), which is as it should be.

However, again problems remain. Reconsider the set $\left\{! p_{1}, p_{1} \Rightarrow ! p_{2}, ! \neg p_{2}\right\}$, but let the ranking now be $p_{1} \Rightarrow ! p_{2}<! \neg p_{2}<! p_{1}$. Let $p_{1} \Rightarrow ! p_{2}$ stand for the doctor's order to wear a scarf when going outside, let $! \neg p_{2}$ stand for your friends' expectation that you don't wear a scarf, and let ! $p_{1}$ represent your sister's wish that you leave the house. Construct the set in $\mathscr{P}_{\mathcal{I}}^{r}(\{\top\}, I)$ - since $I$ remains fully prioritized, there is again just one such set. The minimal element in Satisfiable $_{\mathcal{I}}(\{\top\}, I)$ is $! \neg p_{2}$, and so is added in the first step. The minimal element in Satisfiable $_{\mathcal{I}}\left(\{\top\} \cup\left\{! \neg p_{2}\right\}^{f}, I\right)$, other than $! \neg p_{2}$, is ! $p_{1}$ which therefore gets added in the second step. Nothing is added in the remaining step: $! \neg p_{2}$ and $! p_{1}$ have already been added, and $p_{1} \Rightarrow ! p_{2}$ is not in Satisfiable $\mathcal{I}\left(\{\top\} \cup\left\{! \neg p_{2}, ! p_{1}\right\}^{f}, I\right)$ : though it is triggered by the assumed satisfaction of $! p_{1}$, its consequent is contradicted by the assumed satisfaction of $! \neg p_{2}$. So $\mathscr{P}_{\mathcal{I}}^{\mathrm{r}}(\{\top\}, I)=\left\{\left\{! p_{1}, ! \neg p_{2}\right\}\right\}$. Hence all truth definitions (td-pcd1-8) again makes true $O\left(p_{1} \wedge \neg p_{2} / \top\right)$, so you ought to go out without a scarf, again satisfying the second and third ranking imperatives at the expense of the first, which seems the wrong solution.

\subsection{The fixpoint approach}

To eliminate cases in which the 'reconsidering approach' still adds imperatives that can only be satisfied at the expense of violating a higher-ranking one, a 'fixpoint' approach may seem adequate. Such an approach was first proposed for default reasoning by Brewka \& Eiter [6]. It tests each set that may be considered as preferred to see if it really includes all the elements that should be included: imperatives that are triggered given the facts and the assumed satisfaction of all imperatives in the set, and would be added by Brewka's [4] original method that adds the higher ranking imperatives first. The procedure translates as follows: 
Definition 5 (The fixpoint approach).

Let $\mathcal{I}=\langle I, f, g,<\rangle$ be a prioritized conditional imperative structure, $\Delta$ be a subset of $I$, and $W \subseteq \mathscr{L}_{P L}$ be a set of PL-sentences. Then

$$
\Gamma \in \mathscr{P}_{\mathcal{I}}^{\mathrm{f}}(W, \Delta) \quad \text { iff } \quad \Gamma \in \mathscr{P}_{\mathcal{I}}^{\mathrm{B}}\left(W, \text { Triggered }_{\mathcal{I}}\left(W \cup \Gamma^{f}, \Delta\right)\right) .
$$

To see how this definition works, consider the above set of imperatives $I=$ $\left\{! p_{1}, p_{1} \Rightarrow ! p_{2}, ! \neg p_{2}\right\}$, with the ranking $p_{1} \Rightarrow ! p_{2}<! \neg p_{2}<! p_{1}$. It is immediate that the set $\left\{! p_{1}, ! \neg p_{2}\right\}$ cannot be in $\mathscr{P}_{\mathcal{I}}^{\mathrm{f}}(\{\top\}, I)$ : if we assume all imperatives in this set to be satisfied, then all imperatives are triggered, i.e. $\operatorname{Triggered}_{\mathcal{I}}(\{\top\} \cup$ $\left.\left\{! p_{1}, ! \neg p_{2}\right\}^{f}, I\right)=I$. By Brewka's original method, $\mathscr{P}_{\mathcal{I}}^{\mathrm{B}}(W, I)=\left\{\left\{p_{1} \Rightarrow ! p_{2}, ! p_{1}\right\}\right\}$ : $<$ is already fully prioritized, and for this full prioritization the method adds $p_{1} \Rightarrow ! p_{2}$ in the first step, ! $\neg p_{2}$ cannot be added in the second step since its consequent contradicts the consequent of the previously added $p_{1} \Rightarrow ! p_{2}$, and in the third step $! p_{1}$ is added. So since the considered set $\left\{! p_{1}, ! \neg p_{2}\right\}$ is not in $\mathscr{P}_{\mathcal{I}}^{\mathrm{B}}(W, I)$, it is not a 'fixpoint'. Rather, as may be checked, the only 'fixpoint' in $\mathscr{P}_{\mathcal{I}}^{\mathfrak{f}}(\{\top\}, I)$ is $\left\{p_{1} \Rightarrow ! p_{2}, ! p_{1}\right\}$. For this set all truth definitions (td-pcd1-8) make true $O\left(p_{1} / \top\right)$, but no longer $O\left(p_{1} \wedge \neg p_{2} / \top\right)$. Moreover, truth definitions like $(t d$ $p c d 5-8)$ that allow 'deontic detachment' make true $O\left(p_{1} \wedge p_{2} / \top\right)$. In the given interpretation this means that you must leave the house at your sisters request and wear a scarf, as the doctor ordered you to do in case you go out.

Though the construction now no longer makes true $O\left(p_{1} \wedge \neg p_{2} / \top\right)$, its solution for the example, that determines the set $\left\{p_{1} \Rightarrow ! p_{2}, ! p_{1}\right\}$ as the fixpoint of the set of imperatives $\left\{! p_{1}, p_{1} \Rightarrow ! p_{2}, ! \neg p_{2}\right\}$ with the ranking $p_{1} \Rightarrow ! p_{2}<! \neg p_{2}<! p_{1}$, seems questionable. Though this now includes the doctor's order, you now have no obligation anymore to satisfy the imperative that is second ranking, i.e. your friends' request that you don't wear a scarf; truth definitions $(t d-p c d 4-8)$ even oblige you to violate it by wearing a scarf. Now consider the situation without the third ranking imperative ! $p_{1}$ : it can easily be verified that for a set $I=$ $\left\{p_{1} \Rightarrow ! p_{2}, ! \neg p_{2}\right\}$ the only fixpoint in $\mathscr{P}_{\mathcal{I}}^{\mathrm{f}}(\{\top\}, I)$ is $\left\{! \neg p_{2}\right\}$. So for the reduced set, $(t d-p c d 2)$ makes true $O\left(\neg p_{2} / \top\right)$, i.e. you ought to obey your friends' wish. That the satisfaction of this higher ranking imperative $! \neg p_{2}$ should no longer be obligatory when a lower ranking imperative $! p_{1}$ is added, seems hard to explain. If the ranking is taken seriously, I think one should still satisfy the higher ranking imperatives, regardless of what lower ranking imperatives are added.

However, there is another, perhaps even more severe problem with the fixpoint approach. ${ }^{15}$ Consider a new set of imperatives $\left\{p_{1} \Rightarrow ! p_{2}, !\left(p_{1} \wedge \neg p_{2}\right), ! p_{3}\right\}$, with the ranking $p_{1} \Rightarrow ! p_{2}<!\left(p_{1} \wedge \neg p_{2}\right)<! p_{3}$. For an interpretation, let the first imperative be again the doctor's order to wear a scarf in case you go out, the second one be your friends' request to go out and not wear a scarf, and the third ranking imperative be the wish of your aunt that you write her a letter. Try to find a fixpoint for the default circumstances, i.e. some $\Gamma \in \mathscr{P}_{\mathcal{I}}^{\mathrm{f}}(\{\top\}, I)$ : either $\Gamma$ contains the highest ranking imperative $p_{1} \Rightarrow ! p_{2}$ or it does not. If $\Gamma$ contains it, then $p_{1} \Rightarrow ! p_{2}$ must be in Triggered $_{\mathcal{I}}\left(\{\top\} \cup \Gamma^{f}, I\right)$. It can only be in there if also ! $\left(p_{1} \wedge \neg p_{2}\right)$ is in $\Gamma$, for otherwise $p_{1} \Rightarrow ! p_{2}$ could not be triggered. But no set that

\footnotetext{
$\overline{15}$ Both problems also arise for a new fixpoint approach by John F. Horty in [22].
} 
is constructed by Brewka's method can include both of these imperatives, since their consequents contradict each other. So $\Gamma$ does not contain $p_{1} \Rightarrow ! p_{2}$, contrary to our assumption. So assume $\Gamma$ does not contain $p_{1} \Rightarrow ! p_{2}$. Whatever $\Gamma$ is like, Triggered $_{\mathcal{I}}\left(\{\top\} \cup \Gamma^{f}, I\right)$ includes ! $\left(p_{1} \wedge \neg p_{2}\right)$. By Brewka's method, ! $\left(p_{1} \wedge \neg p_{2}\right)$ will only not be added to the set $\Gamma \in \mathscr{P}_{\mathcal{I}}^{\mathrm{B}}\left(\{\top\}\right.$, $\left.\operatorname{Triggered}_{\mathcal{I}}\left(\{\top\} \cup \Gamma^{f}, I\right)\right)$ if the consequents of previously added imperatives conflict with its consequent - but the only higher ranking imperative is $p_{1} \Rightarrow ! p_{2}$ and we already established that it is not in $\Gamma$. So $!\left(p_{1} \wedge \neg p_{2}\right)$ is in $\Gamma$. But then $p_{1} \Rightarrow ! p_{2}$ is in Triggered $_{\mathcal{I}}\left(\{\top\} \cup \Gamma^{f}, I\right)$, and so is added to $\Gamma$ in the first step of the construction, contrary to the assumption that it is not in $\Gamma$. So there is a reductio ad absurdum for both possible cases, hence there can be no $\Gamma \in \mathscr{P}_{\mathcal{I}}^{\mathrm{f}}(\{T\}, I)$, i.e. there is no fixpoint. So there is also no fixpoint that contains ! $p_{3}$, and hence none of the truth definitions make $O\left(p_{3} / T\right)$ true, and so you do not even have to write to your aunt. But even if the presence of both a higher ranking conditional imperative and a lower ranking, unconditional imperative to violate it poses a problem (why should it? after all, the lower ranking imperative is outranked), it is hard to see why the subject should be left off the hook for all other, completely unrelated obligations. ${ }^{16}$

\subsection{Discussion}

For a discussion of our results so far, let us return to the 'drinking and driving' example from the introduction. Let the three imperatives:

(1) Your mother says: if you drink anything, then don't drive.

(2) Your best friend says: if you go to the party, then you do the driving.

(3) Some acquaintance says: if you go to the party, then have a drink with me.

be represented by a prioritized conditional imperative structure $\mathscr{I}=\langle I, f, g,<\rangle$, where $I=\left\{\left(p_{1} \Rightarrow ! \neg p_{2}, p_{3} \Rightarrow ! p_{2}, p_{3} \Rightarrow ! p_{1}\right\}\right.$ and $p_{1} \Rightarrow ! \neg p_{2}<p_{3} \Rightarrow ! p_{2}<p_{3} \Rightarrow ! p_{1}$. Let the set of facts be $\left\{p_{3}\right\}$, i.e. you go to the party. As we noted, Brewka's original method is not tailored to be directly employed to conditional imperatives. The next three approaches, the naïve, the stepwise and the reconsidering ones, produce $\mathscr{P}_{\mathcal{I}}^{\mathrm{n}}\left(\left\{p_{3}\right\}, I\right)=\mathscr{P}_{\mathcal{I}}^{\mathrm{s}}\left(\left\{p_{3}\right\}, I\right)=\mathscr{P}_{\mathcal{I}}^{\mathrm{r}}\left(\left\{p_{3}\right\}, I\right)=\left\{\left\{p_{3} \Rightarrow ! p_{2}, p_{3} \Rightarrow ! p_{1}\right\}\right\}$, which by all truth definitions ( $t d-p c d 1-8)$ makes true $O\left(p_{1} \wedge p_{2} / p_{3}\right)$, so you ought to drink and drive. The fixpoint approach produces $\mathscr{P}_{\mathcal{I}}^{\mathrm{f}}\left(\left\{p_{3}\right\}, I\right)=\left\{\left\{p_{1} \Rightarrow ! \neg p_{2}, p_{3} \Rightarrow\right.\right.$ $\left.\left.! p_{1}\right\}\right\}$, so all truth definitions make true $O\left(p_{1} / p_{3}\right)$, which means you ought to drink. Truth definition with 'deontic detachment' like ( $t d-p c d 5-8)$ additionally make true $O\left(p_{1} \wedge \neg p_{2} / p_{3}\right)$, so you ought to drink and not drive. But the natural reaction is to ignore the third ranking imperative and drive, as your best friend asked you to do. So it seems we have to look for a different solution.

\footnotetext{
${ }^{16}$ An independent approach by Makinson in [24], which, however, only considers nonprioritized conditionals, also fails in this case: for the default circumstances $T$ it produces the set $\left\{!\left(p_{1} \wedge \neg p_{2}\right), ! p_{3}\right\} . p_{1} \Rightarrow ! p_{2}$ is not considered, since its only 'label' (roughly: a conjunction of the circumstances, the imperatives' antecedents that would trigger* it, and its consequent) is inconsistent (it is $\left.\top \wedge\left(p_{1} \wedge \neg p_{2}\right) \wedge p_{2}\right)$. But it is requires explanation why the agent should not be allowed to obey $p_{1} \Rightarrow ! p_{2}$, rather than having to violate it by satisfying ! $\left(p_{1} \wedge \neg p_{2}\right)$.
} 
Before we do that, I will, however, question again our intuition in this matter. John F. Horty [22] has recently used a structurally similar example to argue for just the opposite, that the solution by the fixpoint approach is correct, i.e. that (in our example) you should drink and not drive. His example is that of three commands, uttered by a colonel, a major and a captain to a soldier, Corporal O'Reilly. The Colonel, who does not like it too warm in the cabin, orders O'Reilly to open the window whenever the heat is turned on. The Major, who is a conservationist, wants O'Reilly to keep the window closed during the winter. And the Captain, who does not like it to be cold, orders O'Reilly to turn the heat on during the winter. The intended representation is again the prioritized conditional imperative structure employed above for the 'drinking and driving' example, where $p_{1}$ now means that the heat is turned on, $p_{2}$ means that the window is closed, and $p_{3}$ means that it is winter. As we have seen, the fixpoint approach yields the preferred subset $\left\{p_{1} \Rightarrow ! \neg p_{2}, p_{3} \Rightarrow ! p_{1}\right\}$, making true $O\left(p_{1} / p_{3}\right)$ with ( $t d-p c d 1-3)$, and $O\left(p_{1} \wedge \neg p_{2} / p_{3}\right)$ with ( $\left.t d-p c d 4-8\right)$, so O'Reilly must turn on the heat and then open the window, and thus violate the Major's order. Horty argues as follows in support of the choice of this set:

"O'Reilly's job is to obey the orders he has been given exactly as they have been issued. If he fails to obey an order issued by an officer without an acceptable excuse, he will be court-martialed. And, let us suppose, there is only one acceptable excuse for failing to obey such an order: that obeying the order would, in the situation, involve disobeying an order issued by an officer of equal or higher rank. (...) So given the set of commands that O'Reilly has been issued, can he, in fact, avoid courtmartial? Yes he can, by (...) obeying the orders issued by the Captain and the Colonel (...). O'Reilly fails to obey the Major's order, but he has an excuse: obeying the Major's order would involve disobeying an order issued by the Colonel."

Horty's principle seems quite acceptable: for each order issued to the agent, the agent may ask herself if obeying the order would involve disobeying an order of a higher ranking officer (then he is excused), and otherwise follow it. The result is a set of imperatives where each imperative is either obeyed, or disobeyed but the disobedience excused. When I nevertheless think the argument is not correct, it is because I think it confuses the status quo and the status quo posterior. Obeying the Major's order does not, in the initial situation, involve disobeying the Colonel's order. Only once O'Reilly follows the Captain's order and turns on the heat, it is true that he must obey the Colonel, open the window and thus violate the Major's order. But this does not mean that he should follow the Captain's order in the first place - as by doing so he brings about a situation in which he is forced, by a higher ranking order, to violate a command from another higher ranking officer. Quite to the contrary, I think that being forced to violate a higher ranking order when obeying a lower ranking one is a case where following the lower one 'involves' such a violation, and so the only order the agent is excused from obeying is the lowest ranking command. 
Another notion seems of importance in such examples: that of coherence, or coherent interpretation, of the imperatives that are accepted by an agent. Suppose I am a trainee at a factory, and over my new workplace there is a large sign: "If the light flashes, press the red button. By order of the Director." On the first day, the foreman tells me "Don't you ever press the red button." A bit later a colleague comes round and tells me "Let's have some fun. Make the light flash. Just short-circuiting it does the job". Obviously I have not been told not to make the light flash. By doing so, I will have to do what the sign tells me and press the red button, and thus violate the foreman's explicit order on my first day. But I can reason as follows: 'Surely, the foreman did not want to contradict the Director's order. But it would amount to a contradiction if the light flashes and I do as he told me and not press the button, though the sign says otherwise. So what the foreman meant was probably this: don't press the button if the light does not flash. So I can safely make the light flash as my colleague told me, and then press the button, thus making everybody happy.' (The consequence of such reasoning would probably be that I lose my job, which might be what my colleague meant by 'fun'.) Such coherent reinterpretation plays an important role in judicial reasoning. But our concern are sets of imperatives that may stem from various sources and contain explicit conflicts. It is the preference ordering that is supposed to take care of arising conflicts. And by closer examination of the situation, if the light flashes, the apparent conflict is resolved since the foreman's order is overridden. Yet that does not mean that I have to accept an obligation to bring about such a situation. If some order is to get me to make the light flash, I think it would have to rank at least as high as the foreman's command, e.g. if my colleague had uttered the imperative in some state of emergency.

Consider finally this variant: suppose that if I am attacked by a man, I must fight him (to defend my life, my family etc.). Furthermore, suppose I have pacifist ideals which include that I must not fight the man. Now you tell me to provoke him, which in the given situation means that he will attack me. Let self-defense rank higher than my ideals, which in turn rank higher than your request. Should I do as you request? By the reasoning advocated by Horty, there is nothing wrong with it: I satisfy your request, defend myself as I must, and though I violate my ideals, I can point out to myself that the requirement to fight back took priority. But I think if I really do follow your advice, I would feel bad. I think this would not just be some irrational regret for having to violate, as I must, my ideals, but true guilt for having been tempted into doing something I should not have done, namely provoking the man: it caused the situation that made me violate my ideals. So I think our intuitions in the 'drinking and driving' example and the other cases have been correct.

\section{$5 \quad$ New Strategies and a New Proposal}

In the face of the difficulties encountered so far, it seems necessary to address the issue of finding an appropriate mechanism for a resolution of conflicts between prioritized conditional imperatives in a more systematic manner. 


\subsection{Deontically Tailored Preferred Subsets}

In the unconditional case, the reason to move from definition $(t d-m 1)$ to $(t d-m 2)$ was that when there are conflicts between imperatives, the former makes true the monadic deontic formula $O \perp$, i.e. the agent ought to do the logically impossible. This result was avoided by considering only maximal sets of imperatives with demands that are collectively consistent, i.e. sets that do not make $O \perp$ true. When faced with the question what dyadic deontic formula should not be true when conflicts are resolved for arbitrary situations $C$, the formula $O(\neg C / C)$ appears to be the dyadic equivalent: it would be weird if a mechanism for conflict resolution results in telling the agent to do something that contradicts the assumed facts. ${ }^{17}$ So to define the set $\mathscr{P}_{\mathcal{I}}(\{C\}, I)$ for a truth definition $(t d$-pcd1$8)$, we can modify Brewka's original method in such a way that it tests, at each step, for each of the constructed subsets, if the corresponding truth-definition $(t d-c d 1-8)$ does not make $O(\neg C / C)$ true for this set. ${ }^{18}$ Formally:

Definition 6 (Deontically Tailored Preferred Subsets).

Let $\mathcal{I}=\langle I, f, g,<\rangle$ be a prioritized conditional imperative structure, and $C \in$ $\mathscr{L}_{P L}$ describe the given situation. Let $(t d-p c d *)$ be any of the truth definitions (td-pcd1-8). Then $\Gamma$ is in the set $\mathscr{P}_{\mathcal{I}}^{*}(\{C\}, I)$ employed by this truth definition iff (i) $\{C\} \nvdash_{P L} \perp$, and (ii) $\Gamma$ is obtained from a full prioritization $\prec$ by defining $\Gamma_{[\prec \downarrow i]}= \begin{cases}\bigcup_{j \prec i} \Gamma_{[\prec \downarrow j]} \cup\{i\} & \text { if }\left\langle\bigcup_{j \prec i} \Gamma_{[\prec \downarrow j]} \cup\{i\}, f, g\right\rangle \not \models O(\neg C / C) \text { by }(t d-c d *), \\ \bigcup_{j \prec i} \Gamma_{[\prec \downarrow j]} & \text { otherwise, }\end{cases}$ for any $i \in I$, and letting $\Gamma=\bigcup_{i \in I} \Gamma_{[\prec \downarrow i]}$.

By this construction, each of the preferred subsets contains a maximal number of the imperatives such that they do not make true $O(\neg C / C)$ for the situation $C$ and the truth definition that is employed, and so the resulting truth definition likewise avoids this truth. Such a construction of the preferred subsets might be considered 'tailored' to the truth definition in question, and any remaining deficiencies might be seen as stemming from the employed truth definition. But this being so, the method reveals a strong bias towards truth definitions that accept 'deontic detachment', and in particular truth definitions ( $t d-p c d 4-8)$ :

Consider the set of imperatives $I=\left\{! p_{1}, p_{1} \Rightarrow ! p_{2}, ! \neg p_{2}\right\}$ with the ranking $! p_{1}<p_{1} \Rightarrow ! p_{2}<! \neg p_{2}$, that was used to refute the 'naïve approach'. As can be easily checked, $\mathscr{P}_{\mathcal{I}}^{*}(\{\top\}, I)=\{I\}$ for all truth definitions $(t d-p c d 1-3)$. So by all these truth definitions, $O\left(p_{1} \wedge \neg p_{2} / \top\right)$ is true. So they commit us to violating the second-ranking imperative, whereas intuitively, the third-ranking imperative should be violated instead. By contrast, all truth definitions ( $t d-p c d 5-8)$, that employ reusable output, and of course likewise $(t d-p c d 4)$ that is equivalent to $(t d-p c d 8)$, handle all given examples exactly as they should be. For the set $I=$

\footnotetext{
$\overline{17}$ For arguments why $O(\neg C / C)$ should be chosen, i.e. for their setting, the 'output' should be consistent with the 'input', rather than the formula $O(\perp / C)$ and thus consistency of output simpliciter, cf. Makinson \& van der Torre [26] p. 158/159.

18 The preferred subsets are thus a choice from the 'maxfamilies' defined in [26].
} 
$\left\{! p_{1}, p_{1} \Rightarrow ! p_{2}, ! \neg p_{2}\right\}$ they produce for both, the ranking $! p_{1}<p_{1} \Rightarrow ! p_{2}<! \neg p_{2}$ that was used to refute the 'naïve approach', and the ranking $p_{1} \Rightarrow ! p_{2}<! p_{1}<! \neg p_{2}$ that was used to refute the stepwise approach, the set $\mathscr{P}_{\mathcal{I}}^{*}(\{\top\}, I)=\left\{\left\{! p_{1}, p_{1} \Rightarrow\right.\right.$ $\left.\left.! p_{2}\right\}\right\}, *=4,5,6,7,8$. Thus they all make true $O\left(p_{1} \wedge p_{2} / \top\right)$, committing us to violate the lowest-ranking imperative only, as it should be for these examples. If the imperatives' ranking is instead $p_{1} \Rightarrow ! p_{2}<! \neg p_{2}<! p_{1}$, that was used to refute both the 'reconsidering' and the 'fixpoint' approaches, then $\mathscr{P}_{\mathcal{I}}^{*}(\{\top\}, I)$ is $\left\{\left\{p_{1} \Rightarrow ! p_{2}, ! \neg p_{2}\right\}\right\}$, making $O\left(\neg p_{2} / \top\right)$ true by all these truth definitions, which thus commit us to satisfying the second ranking imperative, and not to violating it in favor of satisfying the third ranking imperative as these approaches did. Finally the set $I=\left\{p_{1} \Rightarrow ! p_{2}, !\left(p_{1} \wedge \neg p_{2}\right), ! p_{3}\right\}$ with the ranking $p_{1} \Rightarrow ! p_{2}<!\left(p_{1} \wedge\right.$ $\left.\neg p_{2}\right)<! p_{3}$, that was also mishandled by the 'fixpoint approach', produces the set $\mathscr{P}_{\mathcal{I}}^{*}(\{\top\}, I)=\left\{\left\{p_{1} \Rightarrow ! p_{2}, ! p_{3}\right\}\right\}$. So it rejects the second ranking imperative, that commits to violating the higher ranking one, and keeps both others, as it should be. The 'drinking and driving' example is also handled correctly: the set $\left\{p_{1} \Rightarrow ! \neg p_{2}, p_{3} \Rightarrow ! p_{2}, p_{3} \Rightarrow ! p_{1}\right\}$, with the ranking $p_{1} \Rightarrow ! \neg p_{2}<p_{3} \Rightarrow ! p_{2}<p_{3} \Rightarrow ! p_{1}$ produces, for the situation $p_{3}$, the set $\mathscr{P}_{\mathcal{I}}\left(\left\{p_{3}\right\}, I\right)=\left\{\left\{p_{1} \Rightarrow ! \neg p_{2}, p_{3} \Rightarrow ! p_{2}\right\}\right\}$. So the third ranking imperative, that commits the agent to drinking and thus, by observation of the highest ranking imperative, prevents the agent from driving, is disregarded. Instead, the truth definitions make true $O\left(p_{2} / p_{3}\right)$, so the agent must do the driving if she goes to the party, as her best friend asked her to.

Is this the solution to our problems, then? Some uneasiness remains as to the quick way with which definitions (td-pcd1-3) were discharged as insufficient. Why should it not be possible to maintain, as these definitions do, that conditional imperatives only produce an obligation if they are factually triggered, while at the same time maintaining that the above examples should not be resolved the way they are by (td-pcd1-3)? The purpose of a truth definition for the deontic $O$-operator is to find a formal notion of 'ought' that reflects ordinary reasoning, and our intuitions on that matter may differ from our ideas as to what may constitute a good choice from a possibly conflicting set of prioritized conditional imperatives. I will now make a new proposal how to construct the 'preferable subsets', that keeps the positive results without committing us to prefer any of (td-pcd1-8) by virtue of their handling of prioritized imperatives alone.

\subsection{Preferred Maximally Obeyable Subsets}

What made Brewka's approach so successful is that it maximizes the number of higher ranking imperatives in the preferred subsets of a given set of unconditional imperatives: for each 'rank', a maximal number of imperatives are added that can be without making the set's demands inconsistent in the given situation. As was shown, Brewka's approach cannot be directly applied to conditional imperatives, since it makes no sense to test the demands of imperatives for inconsistencies if these imperatives may not be triggered in the same circumstances. Just considering triggered imperatives is also not enough, as was demonstrated for the 'naïve approach'. But if the maximization method is to include imperatives that are not (yet) triggered, then we must look for something else than inconsistency of demands to take the role of a threshold criterion for the maximization process. 
To do so we should ask ourselves why, for the unconditional case, the aim was to find a maximal set of imperatives with demands that are collectively consistent with the situation. I think that by doing so we intend to give the agent directives that can be safely followed. While in the unconditional case this means that the agent can satisfy all the chosen imperatives, the situation is different for conditional imperatives: here an agent can also obey imperatives without necessarily satisfying them. If you tell me to visit you in case I go to Luxembourg next month, I can safely arrange to spend all of next month at home and still do nothing wrong. If we think not so much of imperatives, but of legal regulations, then I can obviously be a law-abiding citizen by simply failing to trigger any legal norm (even though this might imply living alone on an island): whether I do that or boldly trigger some of the regulations' antecedents and then satisfy those I have triggered seems not a question of logic, but of individual choice. So I think the threshold criterion to be used should be that of obeyability: we should maximize the set of imperatives the agent can thus obey, and only stop when the addition of an imperative means that, given the facts, it or some already added imperative, i.e. one that ranks higher or at least as high, can no longer be obeyed, and so will be violated. ${ }^{19}$

For a given set of conditional imperatives $\Delta$ and a set of factual truths $W$, the subsets of imperatives that can be obeyed are described by Obeyable $_{\mathcal{I}}(W, \Delta)$, i.e. they are those subsets $\Gamma \subseteq \Delta$ such that $W \cup \Gamma^{m} \nvdash_{P L} \perp$. To maximize not by collective consistency of demands, but by collective obeyability, Brewka's original approach can therefore be changed as follows:

Definition 7 (Preferred Maximally Obeyable Subsets).

Let $\mathcal{I}=\langle I, f, g,<\rangle$ be a prioritized conditional imperative structure, $\Delta$ be a subset of $I$, and $W \subseteq \mathscr{L}_{P L}$ be a set of PL-sentences. Then $\Gamma \in \mathscr{P}_{\mathcal{I}}^{\circ}(W, \Delta)$ iff (i) $W \nvdash_{P L} \perp$, and (ii) $\Gamma$ is obtained from a full prioritization $\prec$ by defining

$\Gamma_{[\prec \downarrow i]}= \begin{cases}\bigcup_{j \prec i} \Gamma_{[\prec \downarrow j]} \cup\{i\} & \text { if } W \cup\left[\bigcup_{j \prec i} \Gamma_{[\prec \downarrow j]} \cup\{i\}\right]^{m} \nvdash_{P L} \perp, \text { and } \\ \bigcup_{j \prec i} \Gamma_{[\prec \downarrow j]} & \text { otherwise, }\end{cases}$

for any $i \in \Delta$, and letting $\Gamma=\bigcup_{i \in \Delta} \Gamma_{[\prec \downarrow i]}$.

The change from Brewka's original definition is only minute: we test not the demands of the imperatives for consistency, but their materializations. Note that this is a conservative extension of Brewka's method, since for any unconditional imperative $i$ we have $\vdash_{P L} f(i) \leftrightarrow m(i)$. As can easily be seen, the new construction solves all of the previously considered difficulties, regardless of the chosen truth definition for the deontic $O$-operator:

- To refute a direct application of Brewka's original method, we used the set $I=\left\{p_{1} \Rightarrow ! p_{2}, \neg p_{1} \Rightarrow ! \neg p_{2}\right\}$ with no ranking imposed. $m(I)$ is consistent and so $\mathscr{P}_{\mathcal{I}}^{\circ}\left(\left\{p_{1}\right\}, I\right)=\{I\}$, making $O\left(p_{2} / p_{1}\right)$ true for all definitions (td- $\left.p c d 1-8\right)$. So you ought to wear your boots in case you go out, as it should be.

$\overline{19}$ While S. O. Hansson, in [17] p. 200, also advocates a move from 'consistency' to 'obeyability', what is meant there is rather the step from $(t d-m 2)$ to $(t d-d 1)$. 
- To refute the 'naïve approach', we used the set $I=\left\{! p_{1}, p_{1} \Rightarrow ! p_{2}, ! \neg p_{2}\right\}$ with the ranking ! $p_{1}<p_{1} \Rightarrow ! p_{2}<! \neg p_{2}$. Since $I$ is already fully prioritized, the construction produces just one maximally obeyable subset, which is $\left\{! p_{1}, p_{1} \Rightarrow ! p_{2}\right\}$, as its two imperatives get added in the first two steps, and nothing is added in the third since $m(I)$ is inconsistent. All of (td-pcd1-8) make true $O\left(p_{1} / \top\right)$, none makes true the non-intuitive formula $O\left(\neg p_{2} / \top\right)$, and the definitions (td-pcd5-8) that accept 'deontic detachment' make true $O\left(p_{1} \wedge p_{2} / \top\right)$. So you must go out and wear a scarf, which is as it should be.

- To refute the stepwise approach we used $I=\left\{! p_{1}, p_{1} \Rightarrow ! p_{2}, ! \neg p_{2}\right\}$ with the ordering $p_{1} \Rightarrow ! p_{2}<! p_{1}<! \neg p_{2}$. Still $\left.\mathscr{P}_{\mathcal{I}}^{\circ}(\top\}, I\right)=\left\{\left\{! p_{1}, p_{1} \Rightarrow ! p_{2}\right\}\right\}$, so the sentences made true by truth definitions (td-pcd1-8) likewise do not change, and in particular the non-intuitive formula $O\left(\neg p_{2} / \top\right)$ is still false, and definitions (td-pcd5-8) that accept 'deontic detachment' make true $O\left(p_{1} \wedge p_{2} / \top\right)$, so again you must go out and wear a scarf, which is as it should be.

- To refute the reconsidering and the fixpoint approaches we used again the set $\left\{! p_{1}, p_{1} \Rightarrow ! p_{2}, ! \neg p_{2}\right\}$, but the ranking was changed into $p_{1} \Rightarrow ! p_{2}<! \neg p_{2}<! p_{1}$. Now $\left.\mathscr{P}_{\mathcal{I}}^{\circ}(\top\}, I\right)=\left\{\left\{p_{1} \Rightarrow ! p_{2}, ! \neg p_{2}\right\}\right\}$. Truth definitions (td-pcd1-8) make true $O\left(\neg p_{2} / \top\right)$ but not $O\left(p_{1} / \top\right)$ so you must satisfy the second ranking imperative, but not the third ranking imperative, which is as it should be.

- Troublesome for the fixpoint approach was the set $\left\{p_{1} \Rightarrow ! p_{2}, !\left(p_{1} \wedge \neg p_{2}\right), ! p_{3}\right\}$, with the ranking $p_{1} \Rightarrow ! p_{2}<!\left(p_{1} \wedge \neg p_{2}\right)<! p_{3}$ : no fixpoint could be made out in the set and so the approach produced no preferred subset, making everything obligatory. The preferred maximally obeyable subset is $\left\{p_{1} \Rightarrow\right.$ $\left.! p_{2}, ! p_{3}\right\}$, eliminating the second ranking imperative that demands a violation of the first, and making $O\left(p_{3} / \top\right)$ true under all truth definitions (td-pcd1-8), which again is as it should be.

- Finally, consider the 'drinking and driving' example: the set of imperatives $\left\{p_{1} \Rightarrow ! \neg p_{2}, p_{3} \Rightarrow ! p_{2}, p_{3} \Rightarrow ! p_{1}\right\}$ produces, for the situation $p_{3}$, the set of preferred maximally obeyable subsets $\mathscr{P}_{\mathcal{I}}^{\circ}\left(\left\{p_{3}\right\}, I\right)=\left\{\left\{p_{1} \Rightarrow ! \neg p_{2}, p_{3} \Rightarrow ! p_{2}\right\}\right\}$, making true $O\left(p_{2} / p_{3}\right)$ for all truth definitions (td-pcd1-8), so given that I go to the party I must do the driving, which is as it should be.

As could be seen, all truth definitions now produce the 'right' results in the examples used. Moreover, since all truth definitions refer to the same preferred subsets $\mathscr{P}_{\mathcal{I}}^{o}(\{C\}, I)$, it is possible to index the $O$-operators according to the truth definition employed, and e.g. state truths like $O^{1}(A / C) \wedge O^{5}(B / C) \rightarrow$ $O^{7}(A \wedge B / C)$, meaning that if, for any maximal set of imperatives that I can obey in the situation $C$, imperatives are triggered that demand $A$, and that if I satisfy all such triggered imperatives, I will have to do $B$, then obeying a maximal number of imperatives includes having to do $A \wedge B$. It may well be that natural language 'ought-statements' are ambiguous in the face of conditional demands, the discussion in sec. 3 suggested this. If maximal obeyability is accepted as the threshold criterion that limits what norms an agent can be expected to conform to in a given situation, then definition 7 leaves the philosophical logician with maximal freedom as to what deontic operator is chosen. 


\section{Further Research}

\subsection{Benchmark examples for non-prioritized imperatives}

Inevitably there remains further work to do. First of all, it seems worthwhile to consider what happens if the imperatives are not prioritized, in the sense that either there is no ranking between them or that they all have the same priority. It is immediate that for such imperatives, the set of preferred subsets $\mathscr{P}_{\mathcal{I}}^{\circ}(W, \Delta)$ for a prioritized conditional imperative structure $\mathcal{I}=\langle I, f, g,<\rangle$ and a subset of the imperatives $\Delta$, equals $\max _{\subseteq}$ Obeyable $_{\mathcal{I}}(W, \Delta)$, i.e. the preferred subsets are just all the maximally obeyable subsets of $\Delta$, given the facts $W$. There exist a number of benchmark examples for non-prioritized conditional imperatives, given by Makinson in [24], and I list without proof the solutions we obtain for these examples for the $O$-operators defined here.

\begin{tabular}{|c|c|c|c|}
\hline Source and name & Imperatives & Non-truths & Truths \\
\hline $\begin{array}{l}\text { von Wright [?] } \\
\text { window closing }\end{array}$ & $r \Rightarrow ! c, s \Rightarrow ! \neg c$ & $O(c \wedge \neg c / r \wedge s)$ & $O(c \vee \neg c / r \wedge s)$ \\
\hline $\begin{array}{l}\text { Chisholm [7] } \\
\text { help and inform }\end{array}$ & $! h, h \Rightarrow ! i, \neg h \Rightarrow ! \neg i$ & $\begin{array}{l}O(h / \neg h), \\
O(i / \neg h)\end{array}$ & $\begin{array}{l}O(h \wedge i / \top), \\
O(\neg i / \neg h)\end{array}$ \\
\hline $\begin{array}{l}\text { Forrester }[9] \\
\text { gentle murderer }\end{array}$ & $! \neg k, k \Rightarrow ! g$ & $\begin{array}{l}O(g / \top) \\
O(\neg k / k)\end{array}$ & $\begin{array}{l}O(\neg k / \top) \\
O(g / k)\end{array}$ \\
\hline $\begin{array}{l}\text { Belzer [3] } \\
\text { Reykjavik scenario }\end{array}$ & $\begin{array}{l}\text { 1. ! }(\neg r \wedge \neg g), r \Rightarrow ! g, g \Rightarrow ! r \\
\text { 2. ! } \neg r, ! \neg g, r \Rightarrow ! g, g \Rightarrow ! r\end{array}$ & $O(\neg g / r)$ & $O(g / r)$ \\
\hline $\begin{array}{l}\text { Prakken\& Sergot [33] } \\
\text { cigarettes from a killer }\end{array}$ & $! \neg k, ! \neg c, k \Rightarrow ! c$ & $O(\neg k / k)$ & $O(c / k)$ fails! \\
\hline $\begin{array}{l}\text { Prakken\& Sergot [33] } \\
\text { white fence and dog }\end{array}$ & $\begin{array}{l}! \neg f, f \Rightarrow !(f \wedge w), \\
d \Rightarrow !(f \wedge w)\end{array}$ & $\begin{array}{l}O(\neg f / f), \\
O(\neg f / f \wedge d)\end{array}$ & $\begin{array}{l}O(f \wedge w / f) \\
O(f \wedge w / d \wedge f) \\
O(f \wedge w / d) \text { fails! }\end{array}$ \\
\hline $\begin{array}{l}\text { van der Torre }[42] \\
\text { apples and pears }\end{array}$ & $\begin{array}{l}\text { 1. ! }(a \vee p), ! \neg a \\
\text { 2. ! }(a \vee p) \\
\text { 3. } \neg p \Rightarrow ! a, \neg a \Rightarrow ! p\end{array}$ & $O(\neg a / a)$ & $\begin{array}{l}O(\neg a \wedge p / \top), \\
O(\neg a \wedge p / \neg a) \\
O(p / \neg a)^{\mathrm{I}} \\
O(p / \neg a)\end{array}$ \\
\hline $\begin{array}{l}\text { van der Torre }[42] \\
\text { joining paths }\end{array}$ & $! a, ! b$ & $O(a \wedge b / \neg a \vee \neg b)$ & $O(a \vee b / \neg a \vee \neg b)$ \\
\hline $\begin{array}{l}\text { Makinson [24] } \\
\text { Möbius strip }\end{array}$ & $a \Rightarrow ! b, b \Rightarrow ! c, c \Rightarrow ! \neg a$ & $O(\neg a / a)$ & $O(c / a)$ fails! \\
\hline $\begin{array}{l}\text { Makinson [24] } \\
\text { exclusive options }\end{array}$ & $c \Rightarrow !(a \wedge b), \neg c \Rightarrow !(a \wedge \neg b)$ & & $O(a / \top)^{\mathrm{II}}$ \\
\hline
\end{tabular}

I $O$-operators that accept 'circumstantial reasoning' only, i.e. $(t d-p c d 2,4,6,8)$.

II $O$-operators that accept 'reasoning by cases' only, i.e. $(t d-p c d 3,4,7,8)$.

So there are three benchmark examples for which our definitions fail:

In the first one, proposed by Prakken \& Sergot [33] and termed 'cigarettes from a killer', the imperative ! $\neg k$ is intended to mean that you should not kill a certain man, $! \neg c$ means that you should not offer this man a cigarette, and $k \Rightarrow ! c$ means that if you kill the man, you should offer him a cigarette first. Prakken \& Sergot argue that the solution should make true $O(c / k)$, as this applies the imperative that is more specific for the given circumstances, but 
none of the operators provides this result. A similar idea underlies the second example, also proposed by Prakken \& Sergot [33] and termed above 'white fence and dog'. There is a general prohibition of fences $\neg f$ except if there already is one - in that case it should be white, i.e. $f \Rightarrow !(f \wedge w)$ - or if the owner has a dog, in which case the owner should have a white fence, i.e. $d \Rightarrow !(f \wedge w)$. Again, Prakken \& Sergot intend the more specific imperative to be applied in the situation where there is a dog, and so argue that $O(f \wedge w / d)$ should hold. It is true none of the operators defined above includes a 'specificity test', and I do not think that this is a defect. The legal principle lex specialis derogat legi generali is not universally applicable to all sets of norms, in particular if they may stem from various sources, and even in the realm of law it competes with other principles like lex posterior, lex superiori, or standard argument forms like teleological interpretation. But if in the given case the more specific imperative should take priority, we can use a priority ordering that includes $k \Rightarrow ! c<! \neg c$ in case of the first example, and $d \Rightarrow !(f \wedge w)<! \neg f$ in the case of the second. Then all operators $(t d-p c d 1-8)$ make true $O(c / k)$ and $O(f \wedge w / d)$, as intended.

The third example that the truth definitions fail is Makinson's [24] 'Möbius strip': here the set of imperatives is $\{a \Rightarrow ! b, b \Rightarrow ! c, c \Rightarrow ! \neg a\}$. Makinson argues that intuitively, $O(b \wedge c / a)$ should hold. But as is immediate, any maximally obeyable set includes just two of the given imperatives, which does not suffice for the truth of $O(b \wedge c / a)$ for any of $(t d-p c d 1-8)$. The argument why $O(b \wedge c / a)$ ought to be true seems to be that since the consequent of the third imperative $c \Rightarrow ! \neg a$ is false in the supposed situation $a$, the agent cannot do anything about it even if its antecedent becomes true, and so this imperative should not be considered. ${ }^{20}$ But is this argument sound? Even if the consequent is inevitably false, there will be a violation only if its antecedent is (made) true. Certainly, I do not think that the agent should, in such cases, be under an obligation to make the antecedent false - this would introduce a 'deontic contraposition' that, as we saw, is not generally desirable. But that does not mean that the agent should accept an obligation to make the antecedent true. Consider this example: a professor tells a student that next time he sees her, he must have some written paper to present. The student's mother, who is worried about his $\mathrm{PhD}$ not getting finished, wants him to see his professor. The fact is: he does not, and will not, have a written paper. Should he therefore have to go and see his professor? I think that it is entirely up to the agent which of the two imperatives he is going to obey, either attributing higher weight to the explicit order of his professor, or giving priority to alleviating his mother's worries. Similarly, in the case of the Möbius strip, it may be that the agent has reasons to think that she must rather disobey one of the first two imperatives than violate the third. Then the set $\{a \Rightarrow ! b, b \Rightarrow ! c\}$ is not an acceptable choice in the situation $a$, so $O(b \wedge c / a)$ should not be true, and so not providing this truth seems not a defect.

${ }^{20}$ Similarly, Greenspan [12] argues that "it seems that oughts are no longer in force when it is too late to see to it that their objects are fulfilled". 


\subsection{Theorems}

Truth definitions (td-pcd1-8) define when a sentence of the form $O(A / C)$ is true or false with respect to a prioritized conditional structure $\mathcal{I}$ and a situation $C$. So I briefly consider what sentences are theorems, i. e. hold for all such structures, given the usual truth definitions for Boolean operators. It is immediate that for all truth definitions, (DExt), (DM), (DC), (DN) and (DD-R) are theorems. (DD-R) states that there cannot be both an obligation to bring about $A$ and one to bring about $\neg A$ unless the situation $C$ is logically impossible, so our truth definitions succeed in eliminating conflicts. All these theorems are 'monadic' in the sense that the situation $C$ is kept fixed; in fact, they are the $C$-relative equivalents of standard deontic logic $S D L$. More interesting are theorems describing the relations between obligations in different circumstances. Obviously we have

(ExtC) If $\vdash_{P L} C \leftrightarrow D$ then $O(A / C) \leftrightarrow O(A / D)$ is a theorem

for all truth definitions, i.e. for equivalent situations $C$, the obligations do not change. As long as truth definitions are not sensitive to conflicts, e.g. for ( $t d$ $c d 1-8)$, we have 'strengthening of the antecedent', i.e. for these definitions

(SA) $O(A / C) \rightarrow O(A / C \wedge D)$

holds. When only maximally obeyable subsets are considered, i.e. for truth definitions ( $t d-p c d 1-8)$, both (SA) and the weaker 'rational monotonicity' theorem $(\mathrm{RM}) \quad \neg O(\neg D / C) \wedge O(A / C) \rightarrow O(A / C \wedge D)$

are refuted e.g. by a set $I=\left\{!\left(p_{1} \wedge p_{2}\right), !\left(p_{1} \wedge \neg p_{2}\right), p_{2} \Rightarrow \neg p_{1}\right\}$ of equally ranking imperatives: though $O\left(p_{1} / \top\right)$ is true and $O\left(\neg p_{2} / \top\right)$ false, $O\left(p_{1} / p_{2}\right)$ is false. However, for all definitions $(t d-p c d 1-8)$, '(conjunctive) cautious monotonicity'

(CCMon) $O(A \wedge D / C) \rightarrow O(A / C \wedge D)$

holds, which states that if you should to two things and you do one of them, you still have the other one left. ${ }^{21}$ Moreover, truth definitions $(t d-p c d 2,4,6,8)$ validate the 'circumstantial extensionality' rule

(CExt) If $\vdash_{P L} C \rightarrow(A \leftrightarrow B)$ then $O(A / C) \leftrightarrow O(B / C)$ is a theorem

that corresponds to 'circumstantial reasoning'. All definitions that accept 'reasoning by cases', i.e. $(t d-p c d 3,4,7,8)$, make

(Or) $O(A / C) \wedge O(A / D) \rightarrow O(A / C \vee D)$

a theorem. Note that (CExt) and (Or) derive

(Cond) $O(A / C \wedge D) \rightarrow O(D \rightarrow A / C)$,

which in turn derives (Or) in the presence of (DC), and that by adding (CExt) and (Or) we obtain again the system PD (cf. sec. 3). Finally, all definitions with 'deontic detachment', i.e. $(t d-p c d 5,6,7,8)$, make

(Cut) $O(A / C \wedge D) \wedge O(D / C) \rightarrow O(A / C)$

a theorem. (Cut) is derivable given (Cond) (use Cond on the first conjunct $O(A / C \wedge D)$ to obtain $O(D \rightarrow A / C)$, agglomerate with $O(D / C)$, and from $O(D \wedge(D \rightarrow A) / C)$ derive $O(A / C))$, which syntactically mirrors the semantic

${ }^{21}$ This is B. Hansson's [16] theorem (19). 
equivalence of definitions $(t d-p c d 4)$ and $(t d-p c d 8)$. Theoremhood of all of the above theorems for semantics that employ the respective truth definitions is easily proved and left to the reader (cf. my [14] and [15] as well as Makinson \& van der Torre [25] for the general outline). Makinson \& van der Torre's results also suggest that these theorems axiomatically define complete systems of deontic logic with respect to semantics that employ the respective truth definitions $(t d$ $p c d 1-8)$, but this remains a conjecture that further study must corroborate. ${ }^{22}$

\subsection{Questions of representation}

One might wonder if it is always adequate to represent a natural language conditional imperative 'if ... then bring about that _ ' by use of a set $I$ containing an imperative $i$ with a $g(i)$ that formalizes '...' and a $f(i)$ that formalizes '_.'. This is because there is a second possibility: represent the natural language conditional imperative by an unconditional imperative $\ulcorner!(g(i) \rightarrow f(i))\urcorner$. We saw in sec. 3 that this is not generally adequate. But that does not mean that such a representation is not sometimes what is required. Consider the crucial imperatives in the previous examples: perhaps what your mother meant was simply 'don't drink and drive'; perhaps what the doctor meant was 'don't go out without a scarf'; perhaps the Colonel meant to tell O'Reilly not to do both, turn the heat on and keep the window closed; perhaps the sign wanted me to see to it that the light does not flash without the button being pressed, perhaps selfdefense required me to see to it that I am not attacked without fighting back. These interpretations seem not wholly unreasonable, and if they are adequate, then the best representation would be by an imperative $\ulcorner!(g(i) \rightarrow f(i))\urcorner$ instead of $\ulcorner g(i) \Rightarrow ! f(i)\urcorner$. It is easy to see that with such a representation, all of the discussed methods would have resolved these examples.

What then are the conditions that make a representation by an unconditional imperative adequate? One test may be to ask: 'Would bringing about the absence of the antecedent condition count as satisfaction of the imperative?'. Would not drinking, not going out, not turning on the heat, making the light not flash, making the man not attack, count as properly reacting to the imperatives in question? It should be if what the imperatives demand is a material conditional, since then the conditional imperatives in question are equivalent to telling the agent 'either don't drink or don't drive, its your decision', 'either don't go out, or wear a scarf', 'either don't turn on the heat, or open the window', etc. Another test would be to examine if contraposition is acceptable. Can we say that your mother wanted you not to drink if you are going to drive, that the doctor wanted you to stay inside if you are not going to wear a scarf, that the Colonel wanted O'Reilly to turn off the heat if the window is closed, that the sign wants you to make the light not flash if the button is not pressed, that self-defense requires you to make the man not attack if you are not going to fight back? If the proper representation is by imperatives that demand a material conditional, then the answers should be affirmative. I do not think these are easy questions, however, and leave them to the reader to discuss and answer at his or her own discretion.

$\left.\overline{{ }^{22} \text { For }(t d-p c d} 4,8\right)$, completeness of $P D$ is immediate from the results in [14], [15]. 


\subsection{The problem of permission}

The definition of the deontic notion of permission in a context of conditional norms is troublesome. ${ }^{23}$ For monadic deontic logic it is generally accepted to define (weak) permission through the absence of an obligation to the contrary, i.e. $P A={ }_{d f} \neg O \neg A$. This has the additional effect of making $O A \vee P \neg A$ a tautology, and so there are not 'gaps' - any state of affairs is positively or negatively regulated. For dyadic operators, the analogue would be $P(A / C)=_{d f} \neg O(\neg A / C)$. But this leads here to counterintuitive results: consider the set $I=\left\{p_{1} \Rightarrow ! p_{2}\right\}$, with the intended interpretation 'if you go out, wear your boots', and truth definitions $(t d$-pcd $1,2,3,5,6,7)$, i.e. those truth definitions that do not collapse into reasoning about the imperatives' materializations. For all these we have $\mathcal{I} \not \models$ $O\left(p_{1} \rightarrow p_{2} / \top\right)$, and so by the above definition we have $\mathcal{I} \models P\left(p_{1} \wedge \neg p_{2} / \top\right)$. So you are permitted to go out without your boots. There are several proposals that overcome this difficulty. Von Wright, in his re-interpretation of deontic logic as rules for rational norm-giving from [46] onwards, has denied the interdefinability of obligation and permission altogether; his theory has the result that in the absence of explicit permissive norms we only have that $O A$ implies $P A$, i.e. anything permitted is also obligatory. Quite similarly, Makinson \& van der Torre [27] have proposed two definitions of conditional permission that, in the absence of explicit permissive norms, either make it coincide with obligation ('forward permission'), or come quite close to it, by demanding that by forbidding the behavior for the same condition, a conflict would be created for some situation ('backward permission'). All these approaches have the strange result that the less is obligatory, the less is allowed. ${ }^{24}$ But surely one can, in some weak sense, say that given the presence of some (conditional) imperatives, an agent is still free to do $A$ in a situation $C$, without saying that $A$ is also obligatory in this situation. It is perhaps a better solution to define

$$
\mathcal{I}=P(A / C) \text { iff } \exists \Gamma \in \mathscr{P}_{\mathcal{I}}(\{C\}, I): \Gamma^{m} \cup\{C\} \nvdash_{P L} \neg A \text {, }
$$

thus defining $A$ as permissible in a situation $C$ if there is a preferred maximally obeyable subset of the imperatives for which bringing about $A$ does not cause a violation. For operators other than $(t d-p c d 4,8)$, this definition is not 'gapless'. E.g. consider the set $I=\left\{! p_{1}, p_{1} \Rightarrow ! p_{2}\right\}$. For truth definitions that do not accept 'deontic detachment', i.e. $(t d-p c d 1,2,3)$, we have neither $O\left(p_{2} / \top\right)$ nor $P\left(\neg p_{2} / \top\right)$ : though we are not yet under an obligation to bring about $p_{2}$, we are also not permitted to bring about $\neg p_{2}$ and thus make satisfaction of the imperative impossible that ought to be triggered. Or consider conditional imperatives whose

${ }^{23}$ I do not consider here the problem of how permissive norms, or licenses, may be represented. For attempts to use a separate set of 'P-norms' alongside what is here the set of imperatives cf. Alchourrón \& Bulygin [1], von Wright [46], Makinson [24] and Makinson \& van der Torre [27].

${ }^{24}$ Consider the set $I=\left\{p_{1} \Rightarrow ! p_{i} \mid i>1\right\}$, and for an interpretation suppose that I have no obligations in the rest of the world, but am a slave once I go to Australia. By 'forward' or 'backward' permission, $P\left(A / \neg p_{1}\right)$ is false for any $A$, i.e. I am not allowed to do anything if I do not go to Australia, and though $P\left(p_{i} / \top\right)$ holds for backward permission, it is only by virtue of $p_{i}$ being obligatory down under. 
consequent has become impossible to satisfy: for a set $I=\left\{p_{1} \Rightarrow ! p_{2}\right\}$ we do not have $O\left(\neg p_{1} / \neg p_{2}\right)$ for truth definitions other than $\left(t d\right.$-pcd4,8) since $p_{1} \Rightarrow ! p_{2}$ is not triggered in the situation $\neg p_{2}$, but it is also not permitted to trigger it, i.e. $P\left(p_{1} / \neg p_{2}\right)$ is not true. This deontic vagueness may indeed be adequate for such situations. Further study must determine if such a definition does not create counterintuitive results. But it is important to see that as far as reasoning about conditional norms is concerned, the old definitions of permission as the absence of prohibition, obligation as the absence of a permission to the contrary, and prohibition as the absence of permission, do no longer hold.

\section{Conclusion}

Reasoning about obligations when faced with different and possibly conditional imperatives is a part of everyday life. To avoid conflicts, these imperatives may be ordered by priority and then observed according to their respective ranks. The 'drinking and driving' case in the introduction presented an example of such natural reasoning. To provide a formal account is, however, additionally complicated by the fact that there are various and mutually exclusive intuitions about what belongs to the right definition of an 'obligation in the face of conditional imperatives', i.e. the definition of a deontic $O$-operator. Based on similar definitions of operators by Makinson \& van der Torre [25], [26] for their 'input/output logic', but leaving the choice of the 'right' operator to the reader, I presented several proposals in sec. 3 for definitions of a dyadic $O$-operator, namely ( $t d-p c d 1-8$ ). These were dependent on a choice of 'preferred subsets' among a given set of prioritized conditional imperatives. A particularly successful method to identify such subsets, but applying to unconditional imperatives only, was Brewka's [4] definition of 'preferred subtheories' within a theory. In sec. 4 I discussed various approaches that extend this method to conditional imperatives, but these failed to produce satisfactory results for a number of given examples. In sec. 5 I first examined an approach that 'tailors' the choice procedure to the truth definition for the deontic $O$-operator in question, where the only criterion is to avoid the truth of $O(\neg C / C)$ for possible circumstances $C$. Though this finally produced the intended results, it did so for truth definitions ( $t d-p c d 4-8)$ only, whereas counterexamples remained for any of the weaker truth definitions $(t d-p c d 1-3)$. I then argued that the solution is to adapt Brewka's method in such a way that it constructs, instead of maximal subsets of imperatives that are collectively satisfiable by an agent, maximally obeyable subsets of the imperatives. I showed that this new proposal provides adequate solutions to all of the examples, and in particular the 'drinking and driving' example is resolved in a satisfactory fashion for all of the discussed deontic operators. In sec. 6 I demonstrated that the new proposal also includes satisfactory results for benchmark examples developed for non-prioritized conditional imperatives; I presented theorems of a deontic logic based on this proposal (though the question of their completeness had to be left open), and finally I showed that there are problems for the representation of conditional imperatives and difficulties for the definition of a deontic $P$-operator that further philosophical discussion and research must address. 


\section{References}

1. Alchourrón, C. E. and Bulygin, E., "The Expressive Conception of Norms", in [19], 95-124.

2. Alchourrón, C. E. and Makinson, D., "Hierarchies of Regulations and Their Logic", in [19] 125-148.

3. Belzer, M., "Legal Reasoning in 3-D", Proceedings of the First International Conference in Artificial Intelligence and Law, Boston: ACM Press, 1987, 155-163.

4. Brewka, G., "Preferred Subtheories: An Extended Logical Framework for Default Reasoning", in: Sridharan, N. S. (ed.), Proceedings of the Eleventh International Joint Conference on Artificial Intelligence IJCAI-89, Detroit, Michigan, USA, August 20-25, 1989, San Mateo, Calif.: Kaufmann, 1989, 1043-1048.

5. Brewka, G., "Reasoning about Priorities in Default Logic", in: Hayes-Roth, B. and Korf, R. E. (eds.), Proceedings of the 12th National Conference on Artificial Intelligence, Seattle, WA, July 31st - August 4th, 1994, vol. 2, Menlo Park: AAAI Press, 1994, 940-945.

6. Brewka, G. and Eiter, T., "Preferred Answer Sets for Extended Logic Programs", Artificial Intelligence, 109, 1999, 297-356.

7. Chisholm, R. M., "Contrary-to-Duty Imperatives and Deontic Logic", Analysis, 24, 1963, 33-36.

8. Downing, P., "Opposite Conditionals and Deontic Logic", Mind, 63, 1959, 491-502.

9. Forrester, J. W., "Gentle Murder, or the Adverbial Samaritan", Journal of Philosophy, 81, 1984, 193-197.

10. van Fraassen, B., "Values and the Heart's Command", Journal of Philosophy, 70, 1973, 5-19.

11. Goble, L., "A Logic for Deontic Dilemmas", Journal of Applied Logic, 3, 2005, 461-483.

12. Greenspan, P., "Conditional Oughts and Hypothetical Imperatives", Journal of Philosophy, 72, 1975, 259-276.

13. Hansen, J., "Problems and Results for Logics about Imperatives", Journal of Applied Logic, 2, 2004, 39-61.

14. Hansen, J., "Conflicting Imperatives and Dyadic Deontic Logic", Journal of Applied Logic, 3, 2005, 484-511.

15. Hansen, J., "Deontic Logics for Prioritized Imperatives", Artificial Intelligence and Law, 2005, forthcoming.

16. Hansson, B., "An Analysis of Some Deontic Logics", Nôus, 3, 1969, 373-398. Reprinted in [18], 121-147.

17. Hansson, S. O., The Structure of Values and Norms, Cambridge: Cambridge University Press, 2001.

18. Hilpinen, R. (ed.), Deontic Logic: Introductory and Systematic Readings, Dordrecht: Reidel, 1971.

19. Hilpinen, R. (ed.), New Studies in Deontic Logic,Dordrecht: Reidel, 1981.

20. Hofstadter, A. and McKinsey, J. C. C., "On the Logic of Imperatives", Philosophy of Science, 6, 1938, 446-457.

21. Horty, J. F., "Reasoning with Moral Conflicts", Nô̂s, 37, 2003, 557-605.

22. Horty, J. F., "Defaults with Priorities", 2006. Draft version of August 18, 2006,

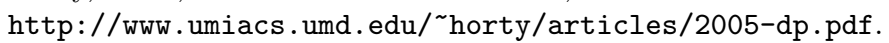

23. Kraus, S., Lehmann, D. and Magidor, M., "Nonmonotonic Reasoning, Preferential Models and Cumulative Logics", Artificial Intelligence, 44, 1990, 167-207. 
24. Makinson, D., "On a Fundamental Problem of Deontic Logic",in: McNamara, P. and Prakken, H. (eds.), Norms, Logics and Information Systems, Amsterdam: IOS, 1999, 29-53.

25. Makinson, D. and van der Torre, L., "Input/Output Logics", Journal of Philosophical Logic, 29, 2000, 383-408.

26. Makinson, D. and van der Torre, L., "Constraints for Input/Output Logics", Journal of Philosophical Logic, 30, 2001, 155-185.

27. Makinson, D. and van der Torre, L., "Permissions from an Input/Output Perspective", Journal of Philosophical Logic, 32, 2003, 391-416.

28. Marek, V. W. and Truszczyński, M., Nonmonotonic Logic. Context-Dependent Reasoning, Berlin: Springer, 1993.

29. Nebel, B., "Belief Revision and Default Reasoning: Syntax-Based Approaches", in: Allen, J. A. and Fikes, R. and Sandewall, E. (eds.), Principles of Knowledge Representation and Reasoning: Proceedings of the Second International Conference, KR '91, Cambridge, MA, April 1991, San Mateo: Morgan Kaufmann, 1991, 417428.

30. Nebel, B., "Syntax-Based Approaches to Belief Revision", in: Gärdenfors, P. (ed.), Belief Revision, Cambridge: Cambridge University Press, 1992, 52-88.

31. Prakken, H., Logical Tools for Modelling Legal Argument, Dordrecht: Kluwer, 1997.

32. Prakken, H. and Sartor, G., "Argument-based Logic Programming with Defeasible Priorities", Journal of Applied Non-classical Logics, 7, 1997, 25-75.

33. Prakken, H. and Sergot, M., "Contrary-to-duty obligations", Studia Logica, 52, 1996, 91-115.

34. Rescher, N., Hypothetical Reasoning, Amsterdam: North-Holland, 1964.

35. Rescher, N., The Logic of Commands, London: Routledge \& Kegan Paul, 1966.

36. Rintanen, J., "Prioritized Autoepistemic Logic", in: MacNish, C. and Pearce, D. and Pereira, L. M., Logics in Artificial Intelligence, European Workshop, JELIA '94, York, September 1994, Proceedings, Berlin: Springer, 1994, 232-246.

37. Ross, W. D., The Right and the Good, Oxford: Clarendon Press, 1930.

38. Ryan, M., "Representing Defaults as Sentences with Reduced Priority", in: Nebel, B. and Rich, C. and Swartout, W. (eds.), Principles of Knowledge Representation and Reasoning: Proceedings of the Third International Conference, KR '92, Cambridge, MA, October 1992, San Mateo: Morgan Kaufmann, 1992, 649-660.

39. Sakama, C. and Inoue, K., "Representing Priorities in Logic Programs", in: Maher, M. (ed.), Joint International Conference and Syposium on Logic Programming JICSLP 1996, Bonn, September 1996, Cambridge: MIT Press, 1996, 82-96.

40. Sosa, E., "The Logic of Imperatives", Theoria, 32, 1966, 224-235.

41. Świrydowicz, K., "Normative Consequence Relation and Consequence Operations on the Language of Dyadic Deontic Logic", Theoria, 60, 1994, 27-47.

42. van der Torre, L., Reasoning About Obligations, Amsterdam: Thesis Publ., 1997.

43. Tröndle, H., "Die Wahlfeststellung", in: Strafgesetzbuch. Leipziger Kommentar, vol. 1, 10th ed., Berlin: Walter de Gruyter, 1985, §1, margin nos. 59-63.

44. von Wright, G. H., "A New System of Deontic Logic", Danish Yearbook of Philosophy, 1, 1961, 173-182. Reprinted in [18], 105-115.

45. von Wright, G. H., An Essay in Deontic Logic and the General Theory of Action, Amsterdam: North Holland, 1968.

46. von Wright, G. H.: "Norms, Truth and Logic", in: von Wright, G. H., Practical Reason: Philosophical Papers vol. I, Oxford: Blackwell, 1983, 130-209.

47. von Wright, G. H.: "Bedingungsnormen, ein Prüfstein für die Normenlogik", in: Krawietz, W., Schelsky, H., Weinberger, O. and Winkler, G. (eds.), Theorie der Normen, Berlin: Duncker \& Humblot, 1984, 447-456. 